\title{
Effect of SEPT6 on the biological behavior of hepatic stellate cells and liver fibrosis in rats and its mechanism
}

\author{
Yuhui Fan ${ }^{1} \cdot$ Zhipeng Du ${ }^{1} \cdot$ Christian J. Steib ${ }^{2} \cdot$ Qiang Ding $^{1} \cdot$ Panpan $\mathrm{Lu}^{1} \cdot$ Dean Tian ${ }^{1} \cdot$ Mei Liu ${ }^{1}$
}

Received: 3 January 2018 / Revised: 25 July 2018 / Accepted: 11 August 2018 / Published online: 12 October 2018

(c) United States \& Canadian Academy of Pathology 2018

\begin{abstract}
Hepatic stellate cells (HSCs) are key effectors during the development of liver fibrosis. Septin 6 (SEPT6) is a highly evolutionarily conserved GTP-binding protein that regulates various cell biological behaviors. The expression and function of SEPT6 in HSCs remain unknown. Here we demonstrate that SEPT6 expression is significantly elevated following the activation of primary rat HSCs, the human hepatic stellate cell line LX-2 and the rat hepatic stellate cell line HSC-T6, as well as in both human and rat fibrotic liver tissue. In vitro, the overexpression of SEPT6 promoted HSCs activation, proliferation, cell cycle progression and migration and inhibited HSCs apoptosis. In contrast, knockdown of SEPT6 exerted the opposite effects on HSCs. Mechanistically, SEPT6 exerted its pro-fibrogenic effect by promoting the expression of TGF- $\beta 1$ and the phosphorylation of Smad2, Smad3, extracellular-signal-regulated kinase, c-Jun NH2-terminal kinase, stress-activated protein kinase-2, and protein kinase B. However, in HSC-T6 cells, blockade of the TGF- $\beta 1 /$ Smad signaling pathway by SB431542 significantly decreased the expression of $\alpha$-smooth muscle actin, cyclin D1, BCL2, and matrix metalloproteinase2 and -9 , which had been enhanced by SEPT6 overexpression. In vivo, adenovirus-mediated SEPT6 inhibition attenuated thioacetamide (TAA)-induced liver fibrosis in rats by decreasing the deposition of the extracellular matrix (ECM). SEPT6 inhibition decreased the proliferation capacity of HSCs and induced apoptosis of HSCs. Collectively, our results reveal that SEPT6 regulates various biological behaviors in HSCs through TGF- $\beta 1 / \mathrm{Smad}$, mitogen-activated protein kinases and phosphatidylinositol-3-kinase/protein kinase B signaling pathways, thus promoting liver fibrosis.
\end{abstract}

\section{Introduction}

Liver fibrosis is a reversible wound-healing response following liver injury that engages a range of cell types and mediators, and results in abnormal and excessive deposition of extracellular matrix (ECM) [1]. The activation of HSCs is a central feature of liver fibrosis; in response to fibrogenic

Electronic supplementary material The online version of this article (https://doi.org/10.1038/s41374-018-0133-5) contains supplementary material, which is available to authorized users.

$\triangle$ Mei Liu

TJliumei@yeah.net

1 Institute of Liver Diseases, Department of Gastroenterology, Tongji Hospital, Tongji Medical College, Huazhong University of Science and Technology, Wuhan, Hubei 430030, P.R. China

2 Department of Medicine II, Liver Center Munich (LCM), Klinikum der Universität München (KUM), Grosshadern, LudwigMaximilians University, Marchioninistrasse 15, 81377 Munich, Germany liver injury, quiescent vitamin A-storing HSCs transdifferentiate into myofibroblasts that are proliferative, contractile, inflammatory and chemotactic, and ECM production is enhanced [2-4]. TGF- $\beta 1$ has been considered the most important cytokine for liver fibrogenesis; once activated, TGF- $\beta 1$ signals are transduced to Smad proteins via cognate receptors, which enhances the transcription of fibrogenic genes, including procollagen I and procollagen III [5]. Mitogen-activated protein kinases (MAPKs) are involved in diverse intracellular signaling pathways; in particular, the activation of extracellular-signal-regulated kinase (ERK), stress-activated protein kinase-2 (p38), and c-Jun NH2-terminal kinase (JNK) appears to be related to liver fibrosis [6-8]. The phosphatidylinositol-3-kinase/ (PI3K/AKT) signaling pathway has also been shown to stimulate HSC proliferation and migration, inhibit HSC apoptosis, and modulate ECM synthesis and degradation [9].

Septins constitute a family of highly evolutionarily conserved GTP-binding proteins that are ubiquitously expressed in all eukaryotes, except for higher plants [10]. 
The first septin was identified in yeast; to date, 13 functional septins (SEPT1 to SEPT12 and SEPT14) and multiple splice variants have been identified [11]. According to the sequence homology of their domain structure, mammalian septins can be divided into four different subgroups named after their founding members: SEPT2, SEPT3, SEPT6, and SEPT7 [12]. Similar to yeast septins, mammalian septins are crucial for multiple processes, including cytokinesis, cytoskeletal organization, cell cycle control, apoptosis, and migration [13-17]. SEPT6 is expressed in all tissues, especially lymphoid and neuron tissues. SEPT6 has been identified as a myeloid/ lymphoid leukemia (MLL) fusion partner in hematological malignancies [18]. Under physiological conditions, SEPT6 is fundamental for development of the nervous system. SEPT6 localizes to microtubules in neuronal dendrites, performs crucial roles in the cytoarchitecture of neurons [19], regulates dendritic outgrowth [20], and is essential for neuronal migration [21]. Biochemical studies have shown that SEPT6 forms a heteromeric complex with SEPT2 and SEPT7 (SEPT2/6/7), and this complex is associated with actin stress fiber, which can regulate the microtubule cytoskeleton during mitosis; thus, this complex might regulate the cell cycle and proliferation [22]. Knockdown of SEPT2, 6, and 7 causes stress fibers to disintegrate and cells to lose polarity via the suppressor of cytokine signaling 7/NCK adaptor protein 1 (SOCS7/ NCK) signaling pathway, resulting in DNA damage and cell cycle arrest [23]. Recent reports have demonstrated that SEPT6 is downregulated in prostate cancer and acts as a tumor suppressor by promoting apoptosis in prostate cancer cells and inhibiting their proliferation, migration, and invasion [24]. However, another study showed that SEPT6 is upregulated by the hepatitis B virus surface antigen gene in HepG2.2.15 and promotes HCC proliferation [25]. This finding implies that SEPT6 plays tissue-specific roles. SEPT4 and SEPT9 have been shown to be decreased following HSCs activation, and the loss of SEPT4 and SEPT9 exacerbates liver fibrosis [26, 27]. We detect the level of the entire septins member in healthy and fibrotic rat liver and find that the SEPT6 is elevated significantly. However, the role of SEPT6 in HSCs and liver fibrosis has not been reported to date.

In this study, we defined and characterized the function of SEPT6 in liver fibrosis for the first time. Our findings reveal that SEPT6 regulates the activation, proliferation, cell cycle progression, apoptosis and migration of HSCs in vitro through the TGF- $\beta 1 /$ Smad, mitogen-activated protein kinases and phosphatidylinositol-3-kinase/protein kinase B signaling pathways, and adenovirus mediated SEPT6 inhibition can block the progression of established fibrosis despite continued liver injury following in vivo administration.

\section{Materials and methods}

\section{Human liver samples}

In total, 40 human liver samples were collected from surgical resections performed at Tongji Hospital (Wuhan, China). Normal liver tissues $(n=20)$ were collected from patients with liver haemangioma and the fibrotic liver tissues $(n=20)$ were obtained from the adjacent liver tissues of patients with liver cancer. All samples were collected with the patients' informed consent and diagnosed by pathologists at Tongji Hospital. This study was performed according to the principles of the Declaration of Helsinki and was approved by approved by the Ethics Committee of Tongji Hospital, Huazhong University of Science and Technology following the ethical and institutional guidelines. The liver tissues were fixed in $4 \%$ paraformaldehyde and embedded in paraffin for the immunohistochemistry staining.

\section{Animal fibrosis models and in vivo adenoviral delivery}

TAA-induced fibrosis models: male Sprague Dawley (SD) rats $(\sim 250 \mathrm{~g}$ weight) obtained from the Department of Experimental Animals of Tongji Medical College were randomly divided into four groups (10 rats per group), including a control group and three model groups. The rats in the model groups were intraperitoneally injected with thioacetamide (TAA; dissolved in physiological saline; 150 $\mathrm{mg} / \mathrm{kg}$ body weight; Ding guo, China) twice a week for 4,8 , or 12 weeks to establish a chronic liver fibrosis model with different degrees of severity. The control rats received an equivalent volume of physiological saline alone at the same time intervals. All rats were raised under pathogen-free conditions with free access to food and water. Then, $24 \mathrm{~h}$ after receiving the final dose, the rats were anaesthetized by $10 \%$ chloral hydrate, and their liver tissues were collected for further analysis. The liver tissues were fixed in $4 \%$ paraformaldehyde, embedded in paraffin and used for the immunohistochemistry and immunofluorescence staining.

In vivo adenoviral delivery: male Sprague Dawley (SD) rats $(\sim 250 \mathrm{~g}$ weights) were randomly divided into four groups (10 rats per group). TAA was injected intraperitoneally $(150 \mathrm{mg} / \mathrm{kg}$, twice a week for 6 weeks) into the rats in groups II, III, and IV, and the rats in group I were treated with the same amount of PBS. Subsequently, the rats were injected with PBS (groups I and II), $5 \times 10^{9}$ pfu control adenovirus vector (AdGFP, group III) or $5 \times 10^{9}$ pfu of SEPT6 shRNA (AdshSEPT6, group IV) via the tail vein twice a week for 2 weeks. The adenoviral vector containing shRNA targeting SEPT6 (AdshSEPT6, targeted sites: 5'-GGGAGATGCTGATTCGAAT-3') and the control 
adenovirus (AdGFP) were purchased from Genechem Co., Ltd. (Shanghai, China). The liver tissues and serum were collected after sacrificing all the rats $72 \mathrm{~h}$ after the final injection. The samples were frozen at $-80^{\circ} \mathrm{C}$ or fixed with $4 \%$ paraformaldehyde for the immunohistochemistry and immunofluorescence staining. RNA and protein were extracted and collected directly.

All animal experiments were performed in accordance with the relevant national and international guidelines. The protocol was approved by the Ethics Committee of Animal Experiments of Tongji Medical College and monitored by the Department of Experimental Animals of the Tongji Medical College, Huazhong University of Science and Technology.

\section{Immunohistochemistry (IHC) staining}

The hepatic specimens were fixed in $4 \%$ paraformaldehyde, embedded in paraffin and sectioned for histology. The sections were dewaxed in xylene, rehydrated in alcohol, and incubated in citric saline for the antigen retrieval. The sections were incubated in $3 \% \mathrm{H}_{2} \mathrm{O}_{2}$ for eliminating the endogenous peroxidase, followed by primary antibodies against SEPT6 (Proteintech, Wuhan, China), $\alpha$-SMA (Abcam, MA, USA), COL1A1 (Proteintech), and matrix metalloprotein (MMP)2 (Proteintech) at $4{ }^{\circ} \mathrm{C}$ overnight. The sections were washed with PBS three times and incubated with the secondary antibody for $60 \mathrm{~min}$ at $37^{\circ} \mathrm{C}$. The antigen-antibody complex described above was visualized by DAB kits (GeneTech, Shanghai, China) according to the manufacturer's instructions. Haematoxylin-eosin (H\&E) staining, Masson trichrome staining and Sirius red staining were used to determine the degree of liver fibrosis. Digital images were taken under an inverted light microscope. $(\times 100, \times 200$ magnification $)$

\section{Immunofluorenscence (IF) staining}

The paraffin-embedded liver tissue sections were dewaxed and rehydrated, and after the antigen retrieval, the sections were permeabilized with Triton X-100 and incubated with primary antibodies against $\alpha$-SMA (Boster, Wuhan, China) and SEPT6 (Proteintech) at $4{ }^{\circ} \mathrm{C}$ overnight. Then, the tissues were washed, incubated with an Alexa Fluor 488conjugated anti-mouse antibody and Alexa Fluor 594 conjugated anti-rabbit antibody (Promoter Biotechnology, Wuhan, China). The nuclei were counterstained with DAPI (Promoter).

The paraffin-embedded liver tissue sections from the adenovirus-treated rats were incubated with a FITCconjugated Ki67 antibody (BD, BioScience, New Jersey, USA) and an anti- $\alpha$-SMA (clone 1A4) cy3-labelled (SigmaAldrich, Hamburg, Germany) antibody to detect the proliferative rate of the HSCs. A FITC-conjugated TUNEL assay (Roche, Mannheim, Germany) was performed with anti- $\alpha$-smooth muscle actin (SMA; clone 1A4) cy3-labelled antibody staining to detect the apoptotic rate in the HSCs as previously described [28]. The nuclei were counterstained with DAPI (Promoter). All immunofluorescence staining assays were performed according to the manufacturer's instructions. Digital images were taken under a fluorescence microscope. ( $\times 100$ magnification)

\section{Cell lines, culture, and treatments}

The primary rat HSCs (R-HSC) were isolated from Sprague Dawley rats (SD, male, 250-300 g) using the Pronase-collagenase perfusion method, subjected to density gradient centrifugation with an OptiPrep gradient, cultured in Dulbecco's modified Eagle's medium (DMEM) with $20 \%$ fetal bovine serum (FBS) (Gibco, Carlsbad, CA, USA) containing penicillin and streptomycin and incubated at $37^{\circ} \mathrm{C}$ with $5 \% \mathrm{CO}_{2}$. The primary rat $\mathrm{HSCs}$ were used for no more than three generations as previously described [29]. The human hepatic stellate cell line LX-2 cells and the rat HSC line HSC-T6 cells were obtained from the Institute of Liver Diseases, Tongji Hospital of Tongji Medical College, Huazhong University of Science and Technology (Wuhan, China) and cultured in DMEM with 10\% FBS.

\section{RNA isolation and quantitative real-time PCR}

Total RNA was extracted from the rat liver tissues, LX-2 and HSC-T6 cells using TRIzol reagent (Invitrogen, Carlsbad, CA, USA) and quantified to $1.0 \mu \mathrm{g} / \mu \mathrm{l}$ by a Thermo Scientific NanoDrop 2000 Spectrophotometer (Thermo Scientific, MA, USA). The first-strand cDNA was synthesized using a PrimeScript reagent kit (Takara, Dalian, China) according to the manufacturer's instructions. SYBR Premix ExTaq (Takara) was used for the real-time PCR analysis of the mRNAs of SEPT6, $\alpha$-SMA, COL1A1, Cyclin D1, Cyclin E1, BAX, BCL2, MMP2, MMP9, and the internal control GAPDH using an ABI StepOne Real-Time PCR System (Applied Biosystem, Carlsbad, CA, USA). The primer sequences are listed in Supplementary Table 1. All experiments were performed in triplicate, and the differences between the samples were determined by the value of $2^{-\Delta \Delta \mathrm{Ct}}$.

\section{Western blot analysis}

The rat liver tissues, primary rat HSCs, LX-2 cells, and HSC-T6 cells were lysed with RIPA buffer containing a phosphatase inhibitor cocktail (Pierce, IL, USA) and PMSF (Goodbio, Wuhan, China), and protein concentration was measured by a BCA protein assay kit (Boster). The total protein in each sample $(50 \mu \mathrm{g})$ was separated by 
SDS-PAGE gel and then transferred to PVDF membranes (Millipore, MA, USA). Then, the membrane was blocked in TBS/Tween-20 (0.05\%) containing 5\% BSA (Boster) for $1 \mathrm{~h}$ at room temperature. Next, the membranes were probed at $4{ }^{\circ} \mathrm{C}$ overnight with the specific primary antibodies listed in Supplementary Table 2. HRP-conjugated IgG anti-rabbit and anti-mouse antibodies (Beyotime, Shanghai, China) were used as secondary antibodies at room temperature for $1 \mathrm{~h}$. The blotting was detected with an ECL assay kit (Peptbio, Wuhan, China) after washing with TBST three times, and all experiments were performed in triplicate. The bands were quantified in greyscale using ImageJ software (NIH, United States).

\section{siRNA, plasmid construction and transfection of LX- 2 cells and HSC-T6 cells}

Small interfering RNA duplexes targeting SEPT6 and scrambled siRNA (NC) were chemically synthesized by RiboBio (Guangzhou, China). The human SEPT6 plasmid, rat SEPT6 plasmid, and control vector (CMV-MCS-EGFPSV40-Neomycin) were purchased from Genechem (Shanghai, China). The plasmids were transfected into LX-2 cells and HSC-T6 cells in a 6-well plate by using Lipofectamine 3000 (Invitrogen) in Opti-MEM (GIBCO) following the manufacturer's protocols. The total RNA and protein were collected after $48 \mathrm{~h}$ or $72 \mathrm{~h}$ to validate the transfection efficiency by real-time PCR and western blot. The siRNA sequences are listed in Supplementary Table 1.

\section{CCK-8 cell proliferation assay}

The proliferation ability of the LX-2 cells and HSC-T6 cells was determined by a Cell Counting Kit-8 (CCK-8) assay. The cells were seeded in 96-well plates at a density of $4 \times$ $10^{3}$ cells per well after transfection with siRNA or plasmid as mentioned above. After cell adherence, the culture medium of each well was replaced with a mixture of $100 \mu \mathrm{l}$ medium and $10 \mu \mathrm{l}$ CCK-8 (Promoter). The absorbance value at $450 \mathrm{~nm}$ wavelength was determined by an ELISA Reader. Cell proliferation was measured at $24 \mathrm{~h}, 48 \mathrm{~h}, 72 \mathrm{~h}$, and $96 \mathrm{~h}$ after transfection.

\section{Cell cycle analysis}

The percentage of LX-2 and HSC-T6 cells in each phase of the cell cycle was measured by flow cytometry. At $72 \mathrm{~h}$ after transfection with the siRNA or plasmid, the cells were washed with cold PBS, trypsinized, collected and fixed in pre-cooled $75 \%$ ethanol at $4{ }^{\circ} \mathrm{C}$ overnight. Then, the cells were washed with cold PBS twice and stained with propidium iodide (PI) staining buffer containing RNase at $37{ }^{\circ} \mathrm{C}$ in the dark for $30 \mathrm{~min}$ according to the manufacturer's instructions. The cell cycle phase was observed and analysed using BD FACS Verse (BD BioScience).

\section{Cell apoptosis analysis}

Similar to the cell cycle analysis, the LX-2 cells and HSCT6 cells were collected $72 \mathrm{~h}$ after the transfection with the siRNA or plasmid. A PE-Annexin-V/7-AAD Cell Apoptosis Kit (BD BioScience) was used to detect the apoptotic cells according to the manufacturer's protocol. The cells were divided into viable cells, early apoptotic cells, advanced apoptotic cells, and dead cells. The percentage of apoptotic cells was observed and analysed using BD FACS Verse (BD BioScience).

\section{Cell wound-healing assay}

The migratory ability of LX-2 cells and HSC-T6 cells was assessed by performing wound-healing assay. The LX-2 cells and HSC-T6 cells were transfected with siRNA or plasmid in 6-well plates; after $24 \mathrm{~h}$, the cells were trypsinized, resuspended and seeded in 12 -well plates $\left(2 \times 10^{5}\right.$ cells/well $)$. After another $24 \mathrm{~h}$, the cell monolayer was scratched using a sterile $200 \mu \mathrm{l}$ micropipette, and the culture medium was replaced with serum-free medium to remove the cell debris. Digital images were taken immediately after scratching and after $24 \mathrm{~h}$ ( $\times 40$ magnification); five images were taken for each sample, and the percentage of the wound-healed area was analysed by using ImageJ software.

\section{Cell transwell migration assay}

The cell migration assay was also performed by using Transwell chambers ( $8 \mu \mathrm{m}$ pore size; Corning, China). LX-2 cells and HSC-T6 cells transfected with siRNA or plasmid for $24 \mathrm{~h}$ were resuspended in $200 \mu \mathrm{l}$ serum-free DMEM and seeded in the upper chamber at a density of $1 \times 10^{4}$ cells per well; the lower chamber was filled with $600 \mu \mathrm{l}$ DMEM supplemented with $20 \%$ FBS as chemoattractant. After incubation for $24 \mathrm{~h}$, the cells that had migrated to the lower chamber of the polycarbonate filter were fixed and stained with crystal violet (Ding guo). Digital images were taken under an inverted light microscope ( $\times 100$ magnification), five fields per well were recorded and the mean number of migrated cells was calculated using ImageJ software.

\section{Statistical analysis}

All experiments were performed in at least triplicate, and the results are presented as the mean \pm SD. The statistical analyses were performed by Student's $t$-test or ANOVA using Prism 5.0 (GraphPad Software, La Jolla, CA, USA). $P<0.05$ was considered statistically significant. 

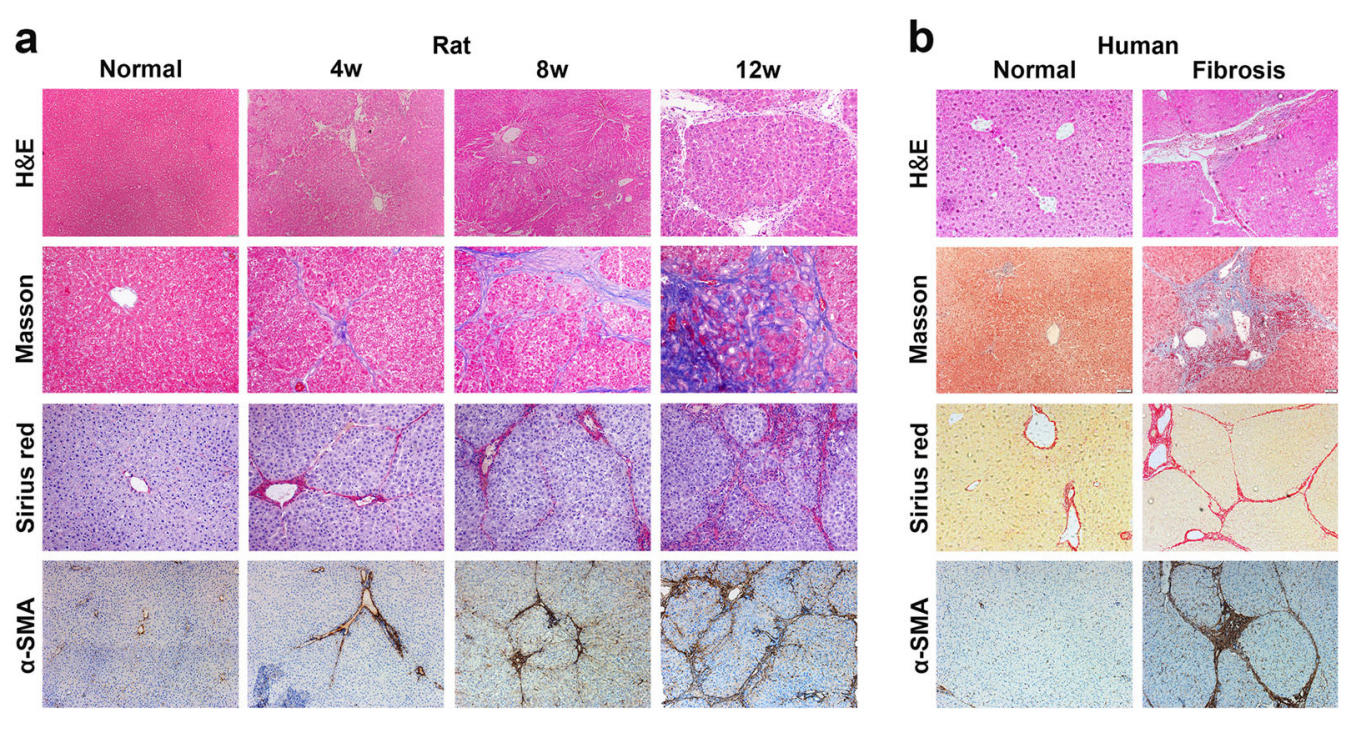

C

d
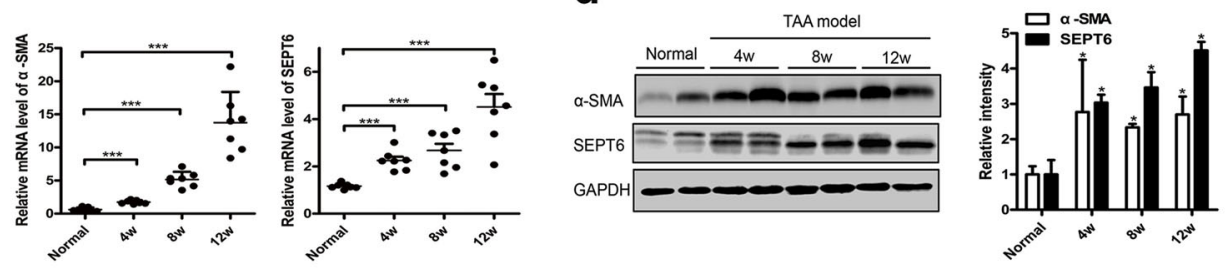

e

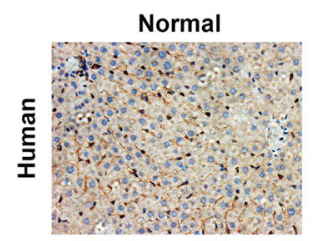

Fibrosis
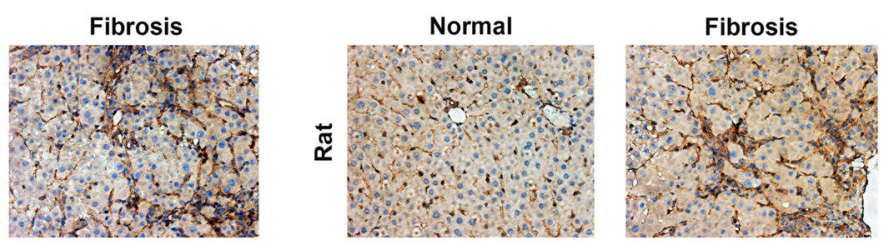

f

a-SMA
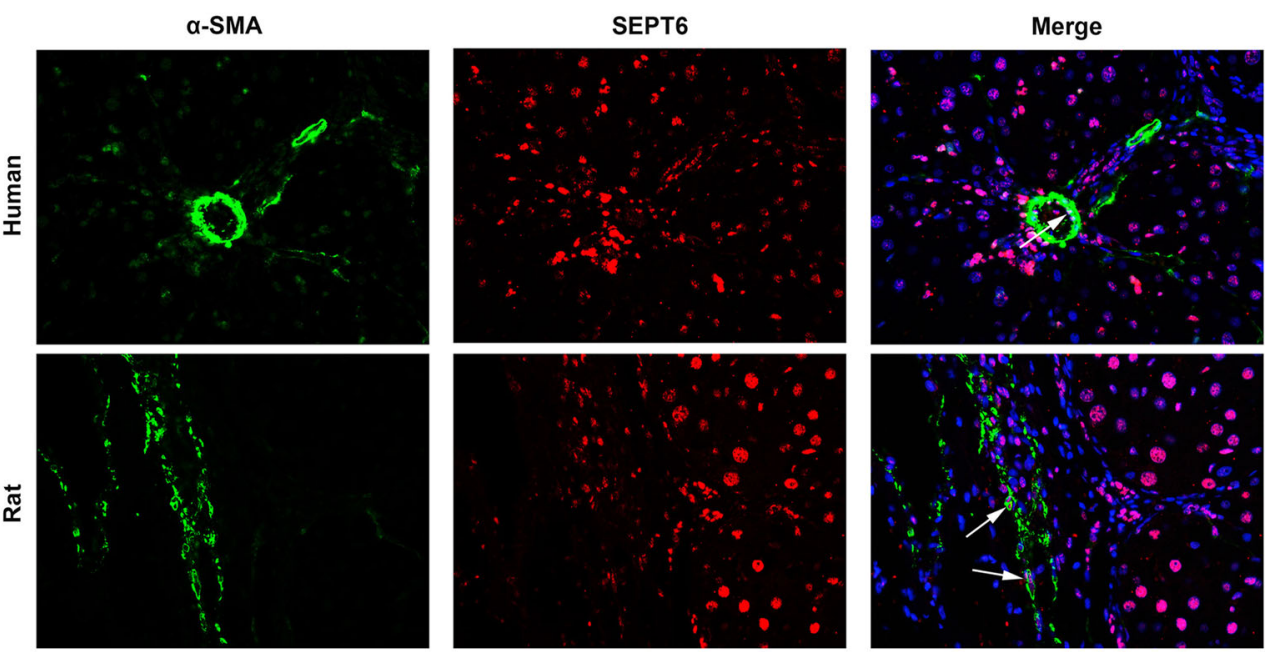

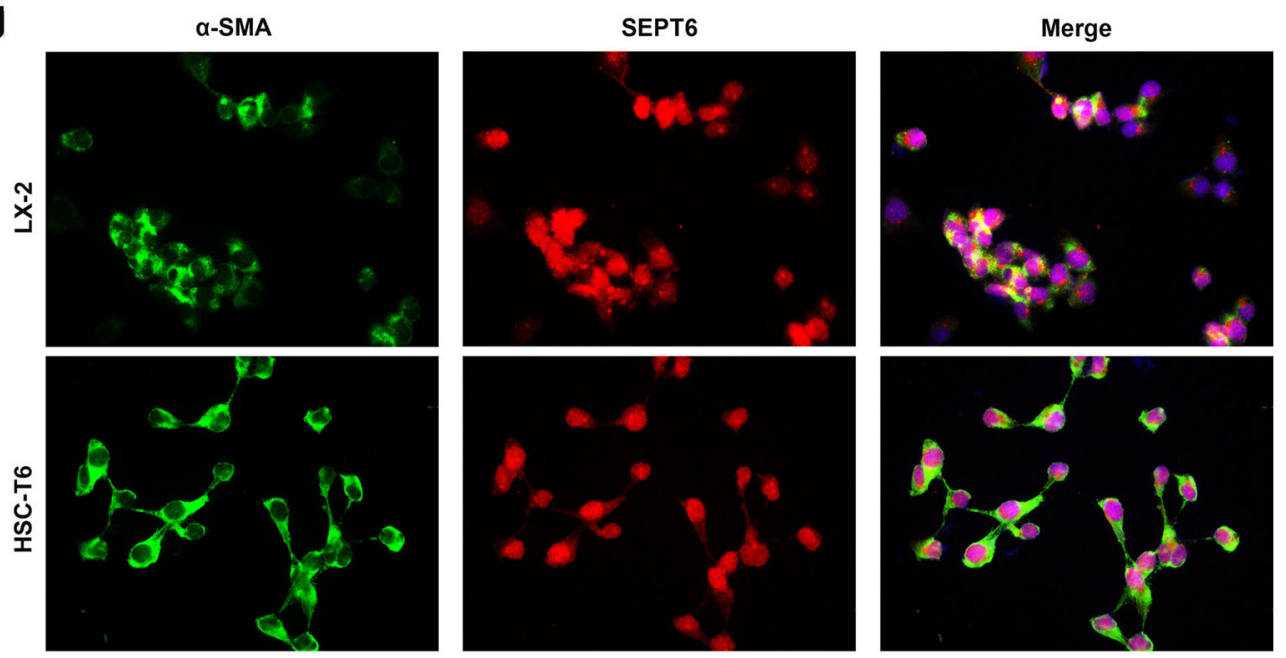

Fig. 1 Increased SEPT6 expression in fibrotic liver tissues. a H\&E, Masson trichrome, Sirius red and $\alpha$-SMA staining in rat and human normal and fibrotic liver tissues. $(\times 100)$. c, $\mathbf{d}$ The mRNA and protein levels of $\alpha$-SMA and SEPT6 in fibrotic livers from TAA-treated rats were detected by real-time PCR $(n=7)$ and western blot $(n=2)$ in each group, respectively. $\mathbf{e}$ The expression of SEPT6 was assessed by immunohistochemistry in human and rat liver tissues. ( $\times 200)$. f Double Immunofluorescence staining of $\alpha$-SMA (green) and SEPT6 (red) in human and rat liver tissues. $(\times 400)$. $\mathbf{g}$ Double Immunofluorescence staining of $\alpha$-SMA (green) and SEPT6 (red) in LX-2 cells and HSC-T6 cells. ( $\times 400)$. GAPDH was used as a loading control. $* P<0.05, * * P<0.01, * * * P<0.001$. Fibrosis vs. Normal by Student's $t$-test or one-way ANOVA

\section{Results}

\section{Expression of SEPT6 is positively correlated with the degree of hepatic fibrosis and activation of HSCs}

To confirm the relationship between SEPT6 and hepatic fibrosis, we established a model of hepatic fibrosis in rats by a TAA intraperitoneal injection. The degree of liver fibrosis was determined by the appearance of the liver (Supplemental Figure S1) and H\&E, Masson trichrome and Sirius red staining and $\alpha$-SMA staining, which showed deformation in the liver architecture and collagen deposition both in rat (Fig. 1a) and human (Fig. 1b) fibrotic tissues. In the TAA-induced liver fibrosis model, we found that the mRNA and protein level of SEPT6 increased significantly as liver fibrosis progressed (Fig. 1c, d).

To explore the distribution and localization of SEPT6 in liver tissues, we performed IHC staining for SEPT6 and double IF staining for $\alpha$-SMA and SEPT6, both in human and rat tissues. The IHC results demonstrated that, both in human and rat tissues, SEPT6 positive staining area in the fibrotic tissue was much more than that of normal tissue, furthermore, SEPT6 was located both in the nucleus and cytoplasm (Fig. 1e). The double IF staining in human and rat tissue showed that SEPT6 was expressed mainly by hepatocyte and was localized mainly in the nucleus, furthermore, co-staining of $\alpha$-SMA and SEPT6 can be observed in HSCs, as the white arrow pointed (Fig. 1f), suggesting that HSCs were a source of SEPT6. The double IF staining for LX-2 cells and HSC-T6 cells demonstrated clearly that SEPT6 was expressed both in the nucleus and cytoplasm, and mainly in the nucleus.

Then, we explored the relationship between SEPT6 and the activation of HSCs in vitro. As previously described, the freshly isolated primary rat HSCs (1 day) were quiescent and activated after 7 days of adherence culture [30]. The mRNA and protein level of $\alpha$-SMA and SEPT6 increased following the activation of rat primary HSCs (Fig. 2a, e). In addition, we treated rat primary HSCs, LX-2 cells, and HSC-T6 cells with PBS or TGF- $\beta 1(10 \mathrm{ng} / \mathrm{ml})$, which is considered the most potent pro-fibrogenic mediator in activating HSCs [31]. As expected, compared with the untreated group, TGF- $\beta 1$ significantly induced the expression of $\alpha$-SMA and SEPT6 (Fig. 2b-d, f-h). These results suggest that SEPT6 may play an important role in hepatic fibrosis and HSC activation.

\section{SEPT6 promotes the activation of HSCs in vitro}

The enhanced level of SEPT6 in HSCs activation and liver fibrogenesis prompted us to further explore the effect of SEPT6 on the biological behavior of HSCs. Two human or rat siRNAs against SEPT6 (si-SEPT6-1 or si-SEPT6-2) were delivered into LX-2 cells or HSC-T6 cells, respectively, to downregulate SEPT6 expression. Furthermore, the human or rat SEPT6 plasmid was delivered into LX-2 cells or HSC-T6 cells to upregulate SEPT6 expression. SEPT6siRNA induced a significant decrease in the SEPT6 mRNA and protein levels in the LX-2 cells (Fig. 3a) and HSC-T6 cells (Fig. 3c); in contrast, the SEPT6 plasmid significantly 
a

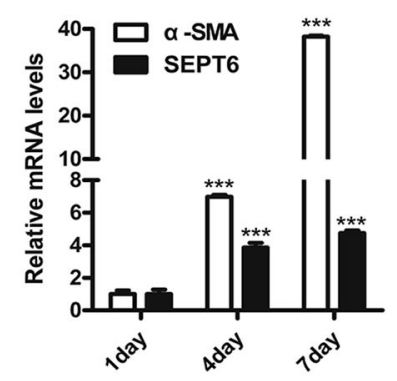

e
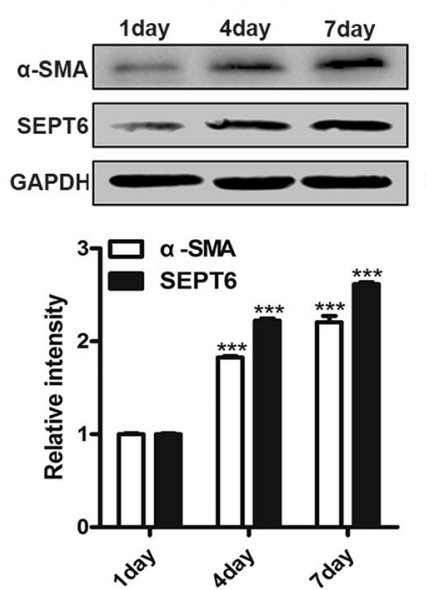

b

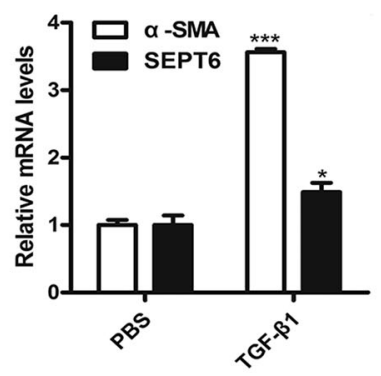

f
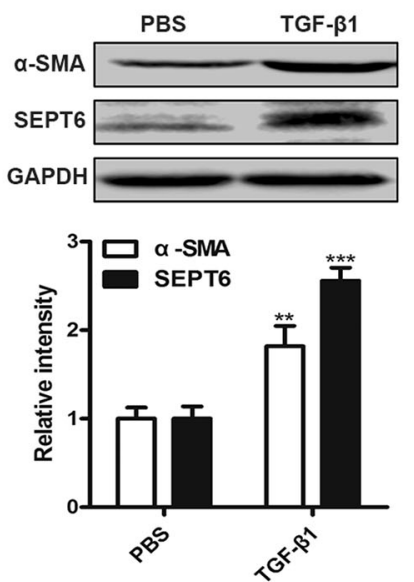

Fig. 2 Increased SEPT6 expression in HSCs activation. a, e Rat primary HSCs (R-HSC) were isolated from normal rats and cultured for 7 days. The level of $\alpha$-SMA and SEPT6 in culture-activated HSCs was detected. $\mathbf{b}$ and $\mathbf{f}$ A total of $2 \times 10^{5}$ freshly isolated rat primary HSCs were seeded in a 6-well plates. Cells were serum-starved for $24 \mathrm{~h}$, and then, PBS or TGF- $\beta 1(10 \mathrm{ng} / \mathrm{ml})$ was added for an additional $24 \mathrm{~h}$. RNA and protein were extracted and the expression levels of $\alpha$-SMA

enhanced the SEPT6 mRNA and protein levels in the LX-2 cells (Fig. 3b) and HSC-T6 cells (Fig. 3d). In the HSC-T6 cells, the SEPT6 knockdown resulted in a significant decrease in $\alpha$-SMA and COL1A1 (Fig. 3e), while the SEPT6 overexpression remarkably increased the $\alpha$-SMA and COL1A1 expression (Fig. 3f). These findings illustrate that SEPT6 can promote HSC activation and collagen synthesis.

\section{SEPT6 promotes the proliferation and cell cycle progression in HSCs in vitro}

Previous studies have shown that the proliferative capacity of activated HSCs contributes to the progression of liver fibrosis. Thus, cell proliferation and the cell cycle were analysed. Compared to NC, LX-2 cells, and HSC-T6 cells transfected with si-SEPT6 (si-SEPT6-1 or si-SEPT6-2) showed a lower proliferative capacity (Fig. 4a, c). In contrast, the upregulation of SEPT6 significantly promoted proliferation in the LX-2 cells (Fig. 4b) and HSC-T6 cells

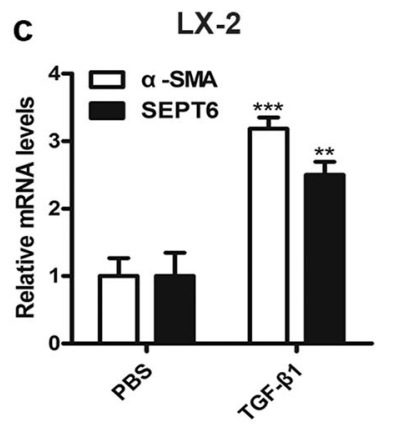

d HSC-T6
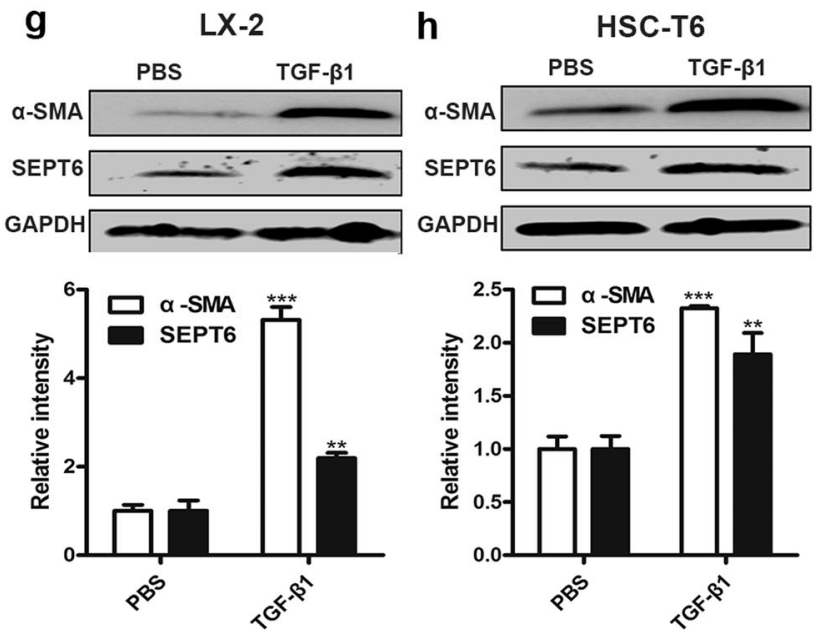

and SEPT6 expression were detected. $\mathbf{c}$ and $\mathbf{g}$ The expression level of $\alpha$-SMA and SEPT6 in LX-2 cells treated with PBS or TGF- $\beta 1$ (10 ng/ $\mathrm{ml})$. $\mathbf{d}$ and $\mathbf{h}$ The expression level of $\alpha$-SMA and SEPT6 in HSC-T6 cells treated with PBS or TGF- $\beta 1(10 \mathrm{ng} / \mathrm{ml})$. GAPDH was used as a loading control. $* P<0.05, * * P<0.01, * * * P<0.001 .4$ day, 7 day vs. 1 day; TGF- $\beta 1$ vs. PBS group by Student's $t$-test or one-way ANOVA

(Fig. 4d). In addition, we performed flow cytometry to evaluate whether SEPT6 knockdown or overexpression could affect the cell cycle progression of LX-2 cells and HSC-T6 cells. The cell cycle analysis showed that the knockdown of SEPT6 increased the G0/G1 phase percentage but decreased $\mathrm{S}$ phase percentage in the LX-2 cells (Fig. 4a) and HSC-T6 cells (Fig. 4c). However, the G2/M phase percentage was increased in the LX-2 cells but decreased in the HSC-T6 cells following the silencing of SEPT6. (Fig. 4a, c) Furthermore, compared with the vector group, we discovered that the enforced expression of SEPT6 decreased the G0/G1 phase percentage and increased the $\mathrm{S}$ phase percentage in both the LX-2 cells (Fig. 4b) and HSC-T6 cells (Fig. 4d). Similarly, the G2/M phase percentage was decreased in the LX-2 cells but increased in the HSC-T6 cells after SEPT6 was enhanced. Furthermore, the real-time PCR and western blot analysis revealed that, in the HSC-T6 cells, knockdown of SEPT6 reduced expression of the Cyclin D1, in contrast, overexpression of SEPT6-enhanced expression of the Cyclin 

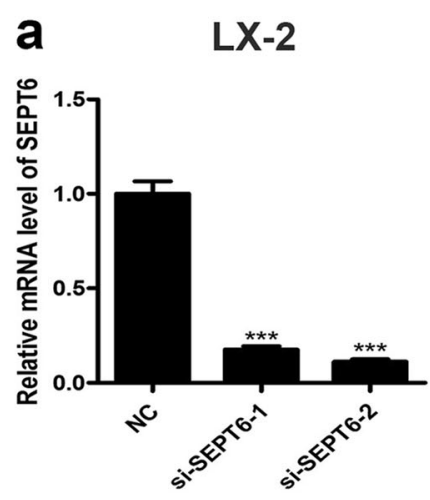

b
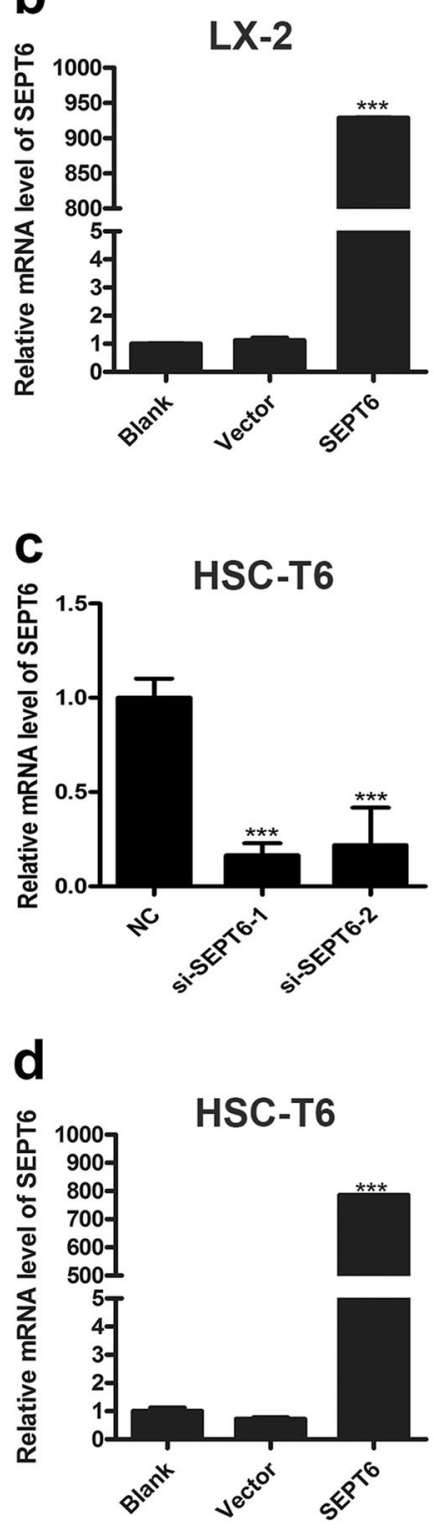
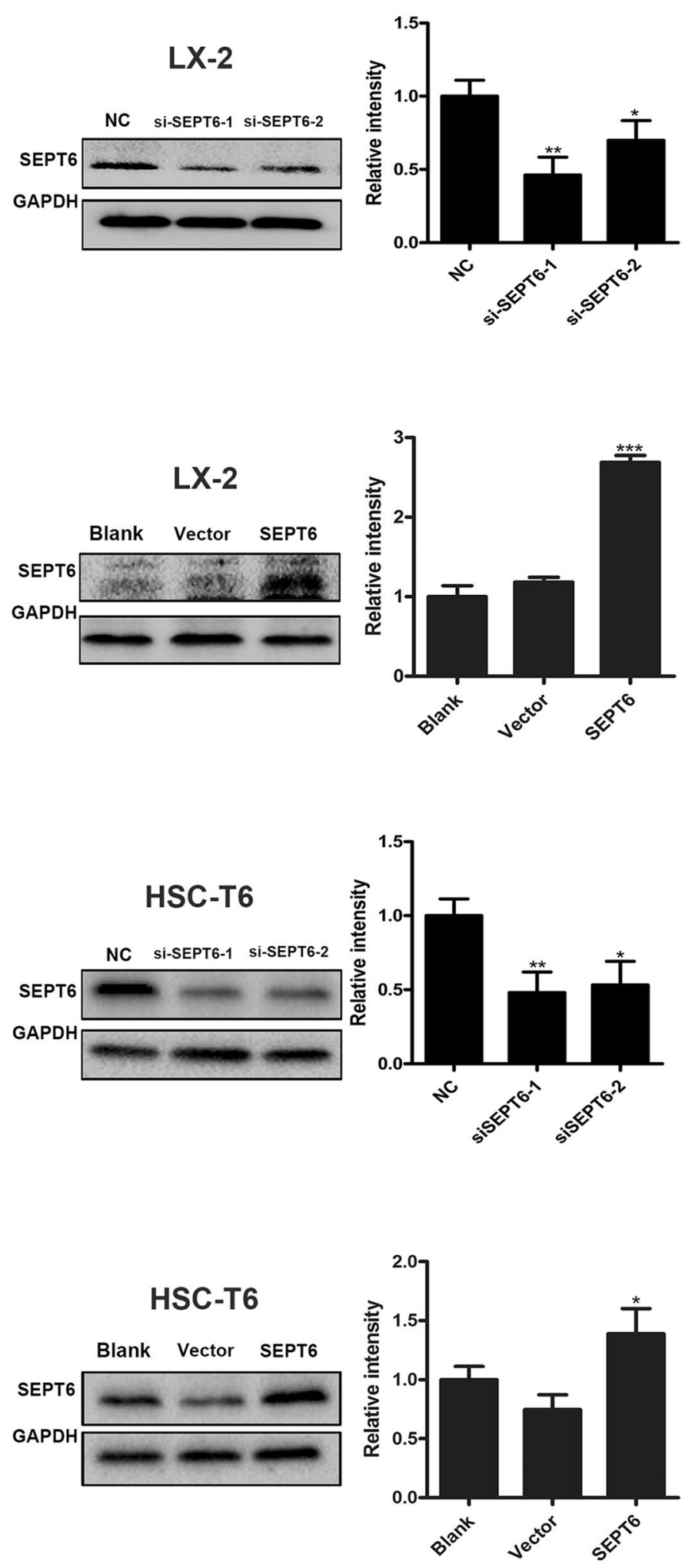

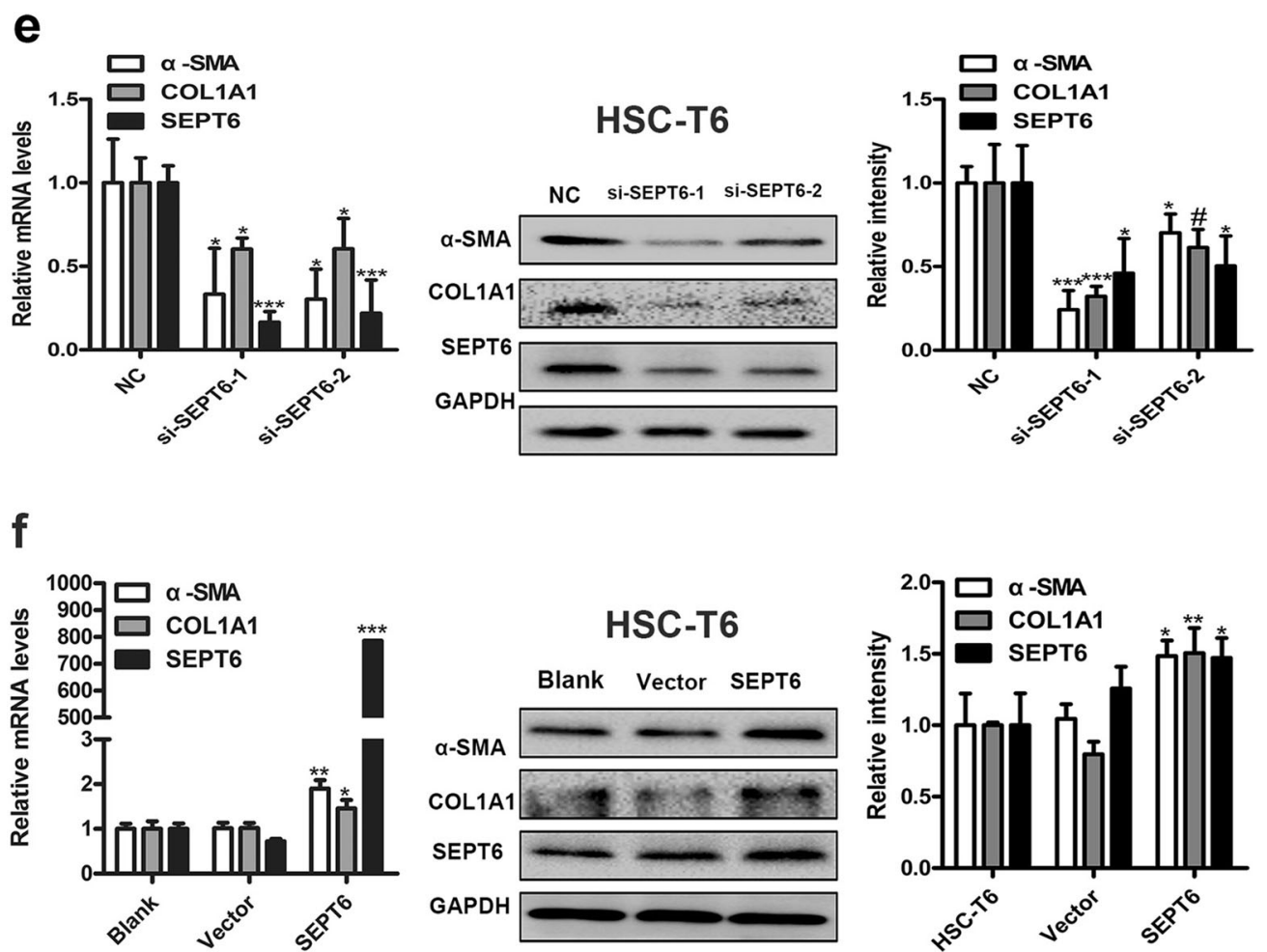

Fig. 3 SEPT6 promotes the activation of HSCs in vitro. a, c The expression level of SEPT6 transfected with siRNA in LX-2 cells a and HSC-T6 cells $\mathbf{c}$ was detected. b, d The expression level of SEPT6 transfected with SEPT6 plasmid in LX-2 cells b and HSC-T6 cells d was detected. $\mathbf{e}$ and $\mathbf{f}$ The expression level of $\alpha$-SMA and COL1A1 were analysed after SEPT6 expression was silenced $\mathbf{e}$ or enhanced $\mathbf{f}$ in HSC-T6 cells. GAPDH was used as a loading control. $* P<0.05, * * P$ $<0.01, * * * P<0.001$. siRNA vs. NC; SEPT6 vs. vector by Student's $t$ test

observed in the LX-2 cells (Fig. 5b, 20.19 vs. 11.45\%) and HSC-T6 cells (Fig. 5d, 18.43 vs. 6.05\%) cells transfected with the SEPT6 plasmid. Further, we performed real-time PCR and western blot to explore whether SEPT6 could regulate the expression of the cell apoptosis-related proteins BAX and BCL2 in HSC-T6 cells. SEPT6 inhibition significantly increased the expression of $\mathrm{BAX}$ and decreased the expression of BCL2 in the HSC-T6 cells (Fig. 5e). Correspondingly, the overexpression of SEPT6 in the HSCT6 cells showed the opposite result (Fig. 5f). Taken together, SEPT6 may play a role in inhibiting apoptosis in HSCs in vitro.

\section{SEPT6 promotes the migration in HSCs in vitro}

Previous studies have indicated that activated HSCs have the ability to migrate to injured sites, and promote the progression of liver fibrosis [33]. Therefore, to confirm the role of SEPT6 in HSC migration, two assays were performed in our study. In the wound-healing assay, compared to the NC group, the knockdown of SEPT6 obviously attenuated the migration ability of the LX-2 cells (Fig. 6a) and HSC-T6 cells (Fig. 6c). In contrast, the overexpression

obvious decrease in the number of early apoptotic cells was 
Fig. 4 SEPT6 promotes proliferation and cell cycle progression in HSCs in vitro. ad Cell proliferation rates were determined by a CCK-8 assay and the percentages of cell cycle distributions were analysed by flow cytometry after SEPT6 expression was silenced in LX-2 cells a and HSC-T6 cells $\mathbf{c}$ or SEPT6 expression was enhanced in LX-2 cells b and HSC-T6 cells d. The absorbance at 450 $\mathrm{nm}$ was determined at the specified time point. e and $\mathbf{f}$ The expression of Cyclin D1 was analysed by real-time PCR and western blot after SEPT6 expression was silenced $\mathbf{e}$ or enhanced $\mathbf{f}$ in HSC-T6 cells. GAPDH was used as a loading control. $* P<0.05, * * P<0.01$, $* * * P<0.001$. siRNA vs. NC; SEPT6 vs. vector by Student's $t$-test or one-way ANOVA a
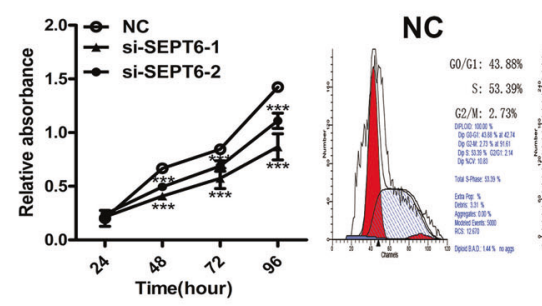

LX-2
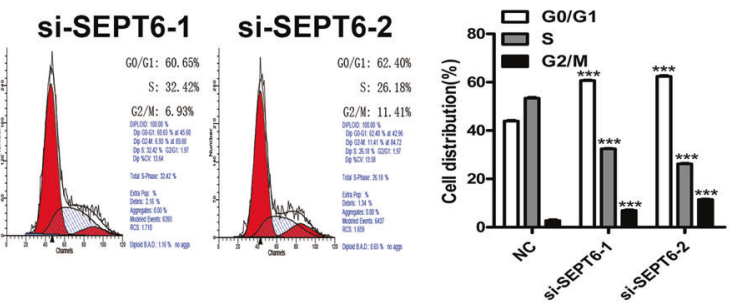

b

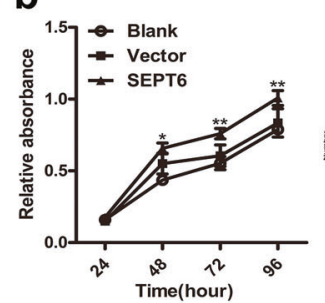

Blank

C
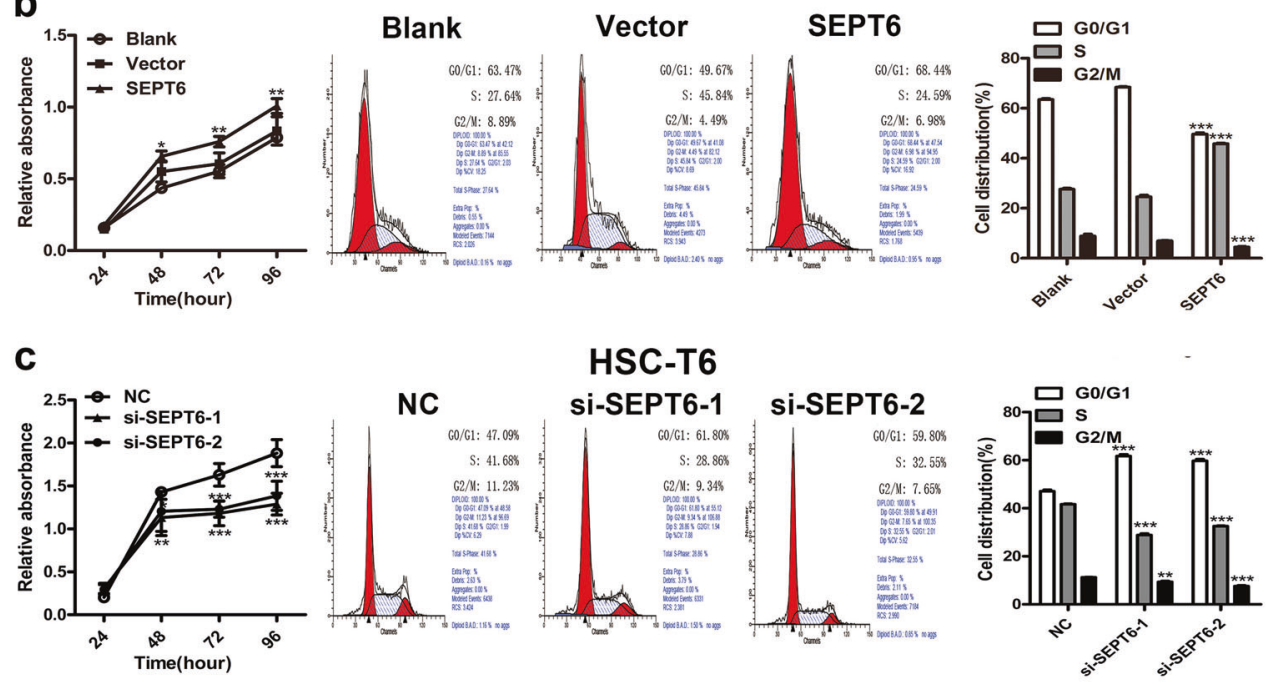

HSC-T6
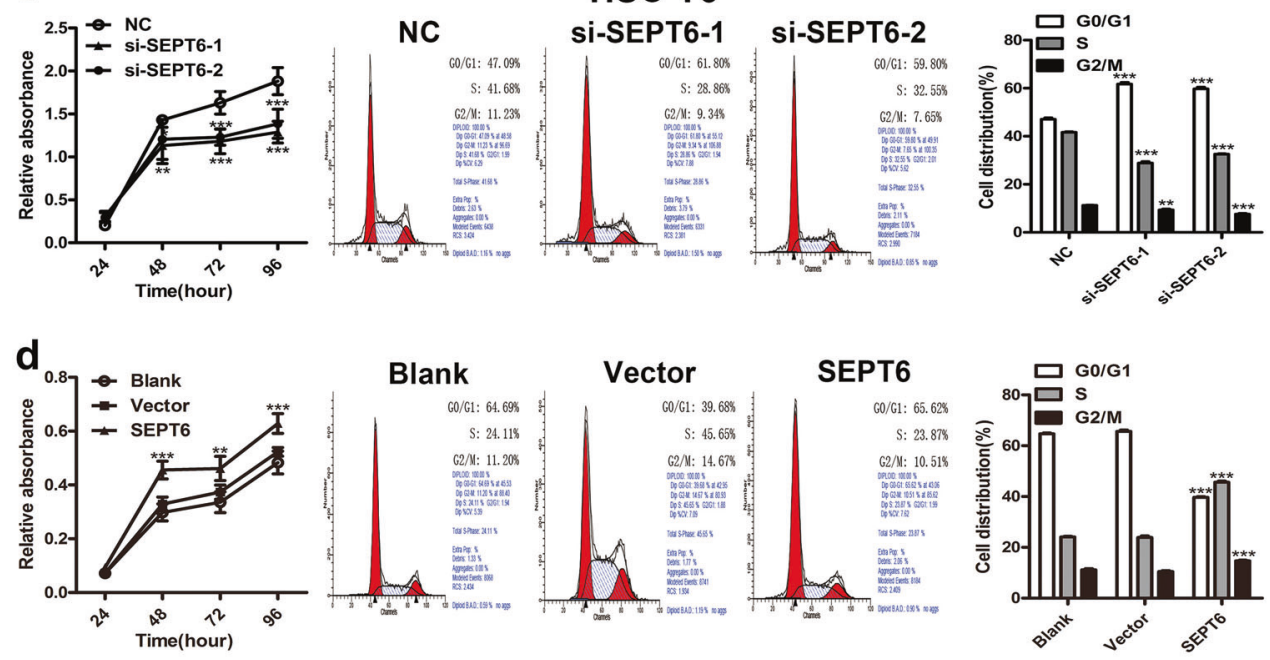

e

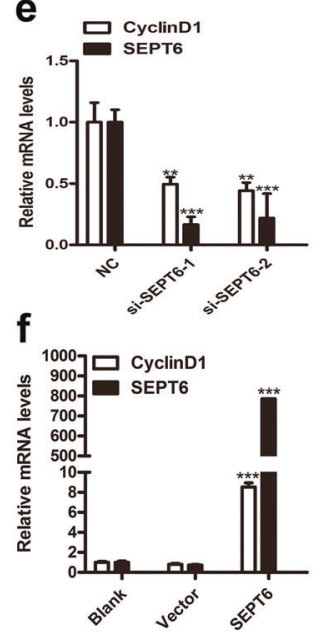

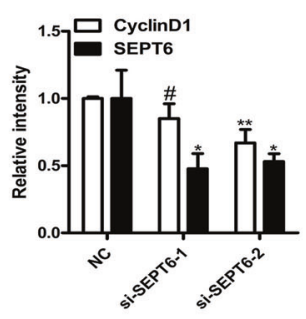
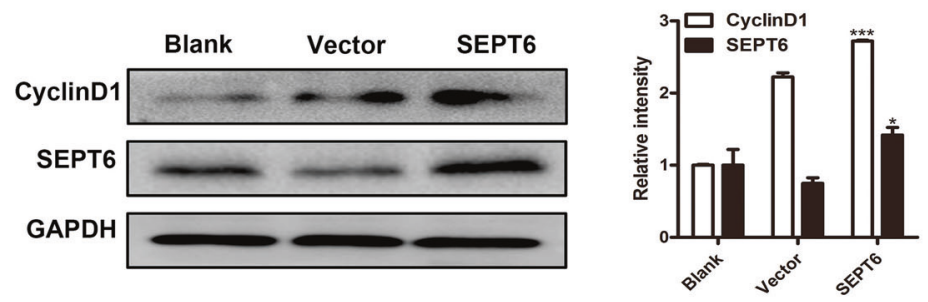

of SEPT6 evidently enhanced the migration capacity of the LX-2 cells (Fig. 6b) and HSC-T6 cells (Fig. 6d). Subsequently, a Transwell assay was performed to further confirm the above result. Consistently, compared to the NC group, the knockdown of SEPT6 significantly decreased the number of cells that penetrated the chamber membrane in 
LX-2 cells (Supplemental Figure S2a) and HSC-T6 cells (Supplemental Figure S2c). Furthermore, the overexpression of SEPT6 significantly enhanced the migratory ability of the LX-2 cells (Supplemental Figure S2b) and HSC-T6 cells (Supplemental Figure S2d); compared with the cells in the vector group, more cells migrated through a

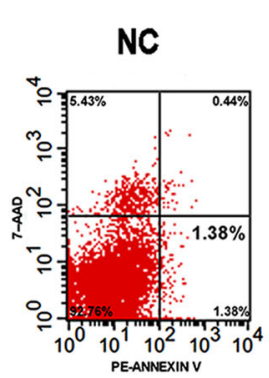

b

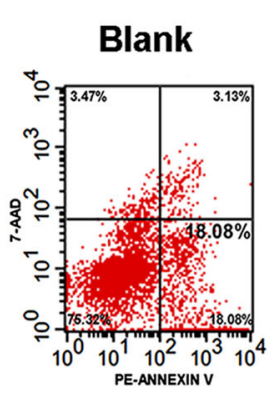

C

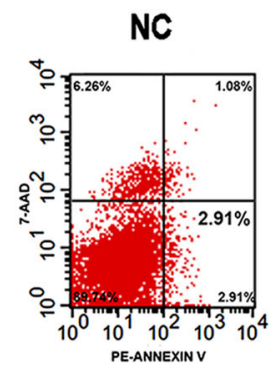

d

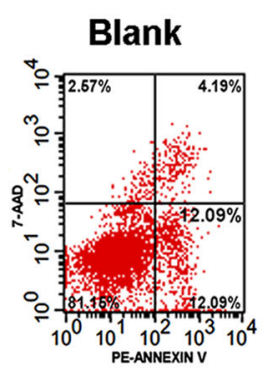

LX-2
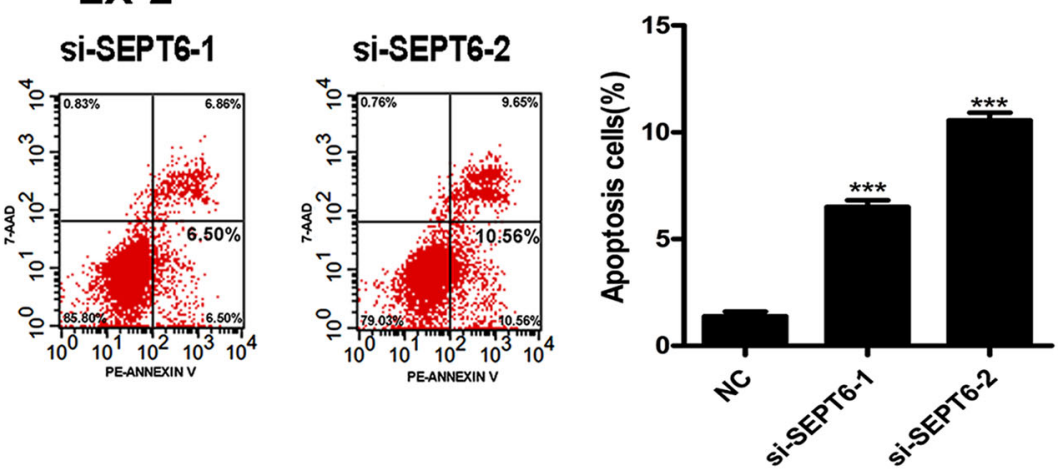

\section{LX-2}

Vector
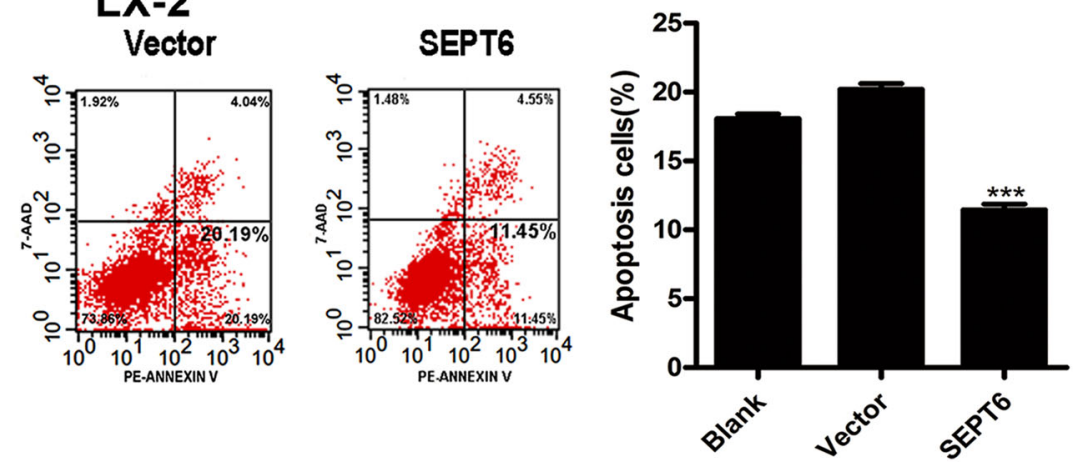

\section{HSC-T6}
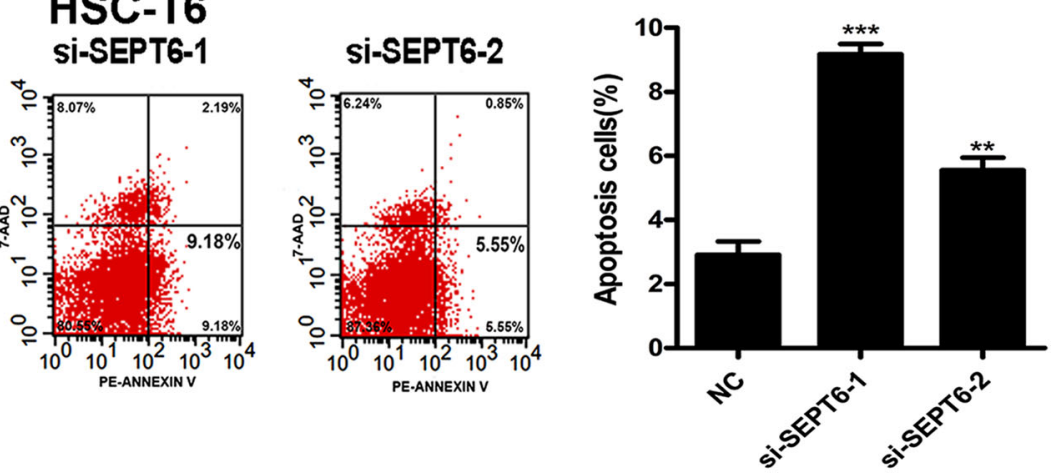

HSC-T6 Vector

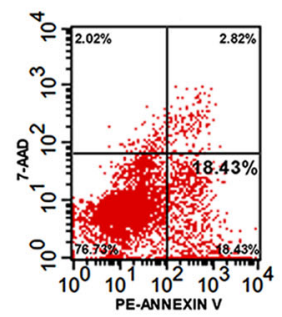

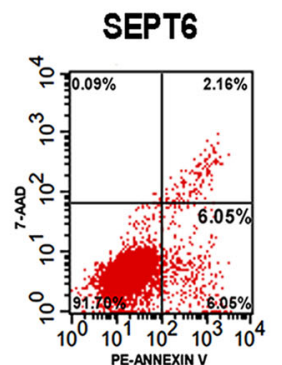



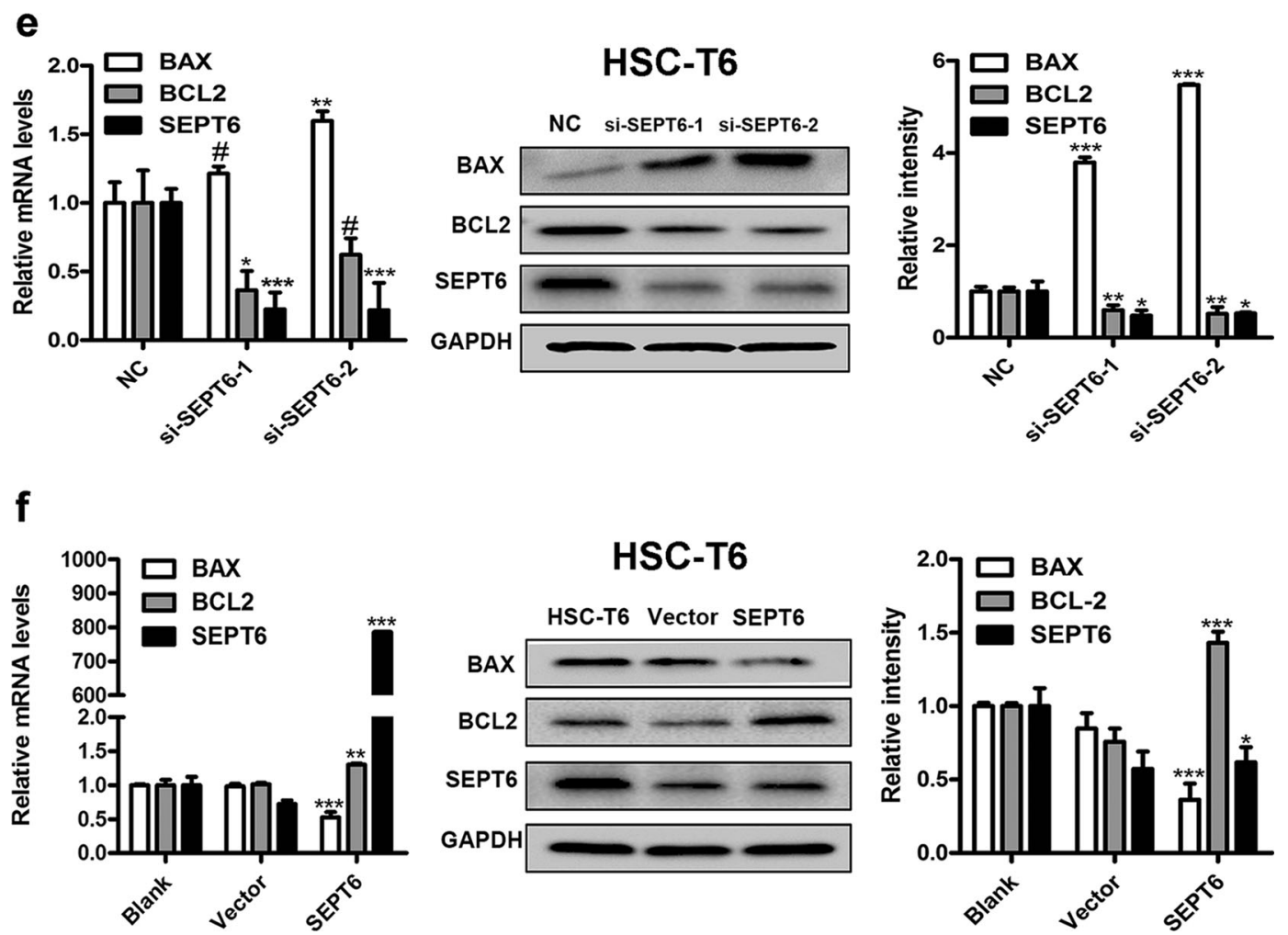

Fig. 5 SEPT6 inhibits apoptosis in HSCs in vitro. a-d Cell apoptosis rates were measured by flow cytometry using PE-labelled Annexin-V/7-AAD staining after SEPT6 expression was silenced in LX-2 cells a and HSC-T6 cells $\mathbf{c}$ or SEPT6 expression was enhanced in LX-2 cells b and HSC-T6 cells $\mathbf{d}$. Representative images of three independent experiments are shown. e, f Genes related to apoptosis including BAX and BCL2 were analysed by real-time PCR and western blot after SEPT6 expression was silenced $\mathbf{e}$ or enhanced $\mathbf{f}$ in HSC-T6 cells. GAPDH was used as a loading control. $* P<0.05, * * P<0.01, * * * P<0.001$. siRNA vs. NC; SEPT6 vs. vector by Student's $t$-test

the pores. Mechanistically, the real-time PCR and western blot revealed that MMP2 and MMP9 were positively regulated by SEPT6 in the HSC-T6 cells (Fig. 6e, f). Taken together, SEPT6 may promote HSC migration in vitro.

\section{SEPT6 induces activation of the TGF- $\beta 1 / \mathrm{Smad}$ signaling pathway in HSC-T6 cells}

The canonical TGF- $\beta 1 /$ Smad signaling pathway has been demonstrated to regulate biological behavior in HSCs in liver fibrosis [34]. Therefore, we examined whether TGF$\beta 1 /$ Smad signaling pathway is involved in SEPT6-driven HSC activation. In our study, the knockdown of SEPT6 in HSC-T6 cells decreased the expression of TGF- $\beta 1$ and the phosphorylation of Smad2 and Smad3 (Fig. 7a). In contrast, the upregulation of SEPT6 significantly enhanced the expression of TGF- $\beta 1$ and the phosphorylation of Smad2 and Smad3 (Fig. 7b). However, the level of total Smad2, Smad3, and Smad4 remained unchanged regardless of whether SEPT6 was silenced or enhanced (Fig. 7a, b). Furthermore, we found that the selective TGF- $\beta$ receptor inhibitor, SB431542, significantly decreased SEPT6induced overexpression of p-Smad2, p-Smad3, $\alpha$-SMA,
Cyclin D1, BCL2, MMP2, and MMP9 in HSC-T6 cells; in contrast, SB431542 increased BAX expression which was initially decreased by SEPT6 overexpression. (Supplemental Figure S3) Interestingly, as shown in Fig. 2, the mRNA and protein level of SEPT6 in HSCs was significantly upregulated following the TGF- $\beta 1$ stimulation. Collectively, our data suggest that SEPT6 activates the TGF- $\beta 1 /$ Smad signaling pathway in liver fibrosis, and a positive feedback loop might exist.

\section{SEPT6 induces the activation of the MAPK and PI3K/ AKT signaling pathways in HSC-T6 cells}

Numerous studies have demonstrated that the MAPK and PI3K/AKT signaling pathways are involved in several physiological behavior of HSCs, including activation, proliferation, differentiation, apoptosis, and migration [9, 35]. To fully understand the promoting effect of SEPT6 in liver fibrosis, we further investigated the effect of SEPT6 on the MAPK and PI3K/AKT signaling pathways in HSC-T6 cells. The results showed that compared to the NC group, the phosphorylation of ERK, JNK, p38, and AKT was blocked in HSC-T6 cells treated with SEPT6-siRNA 
(Fig. 7c). In contrast, the phosphorylation of ERK, JNK, p38, and AKT was significantly enhanced in the HSC-T6 cells following the enhancement of SEPT6 expression by the plasmid transfection (Fig. 7d). However, the expression of total ERK, JNK, p38, and AKT was not significantly influenced by the overexpression or knockdown of SEPT6 a

Oh

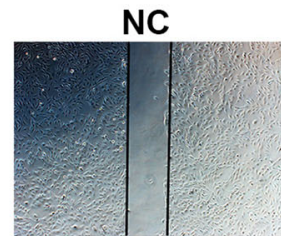

$24 h$

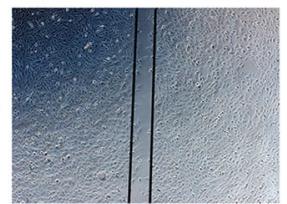

\section{LX-2}
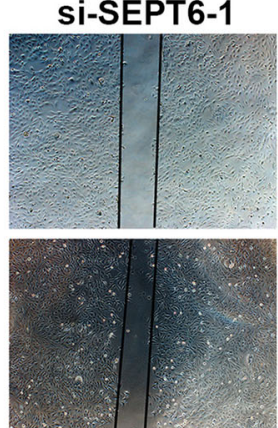

LX-2

b

Oh

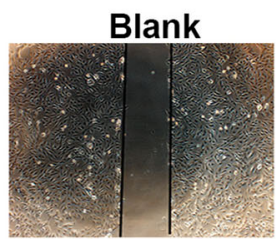

$24 \mathrm{~h}$

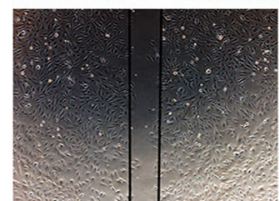

C
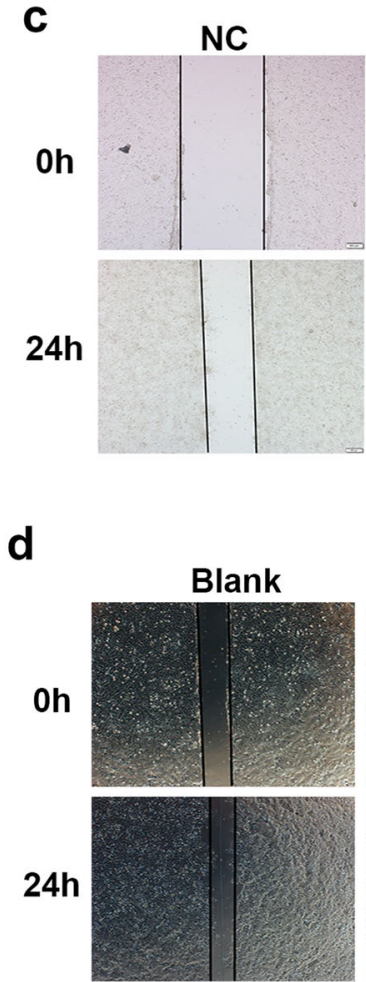

Vector

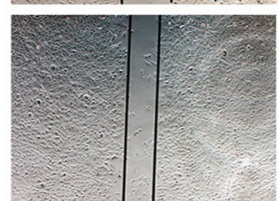

HSC-T6
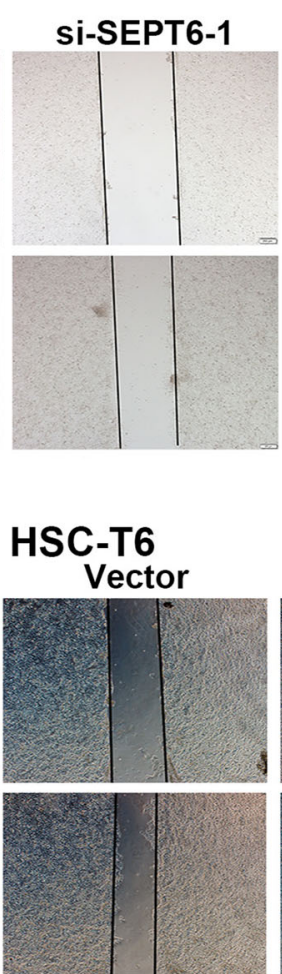

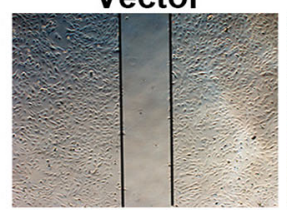

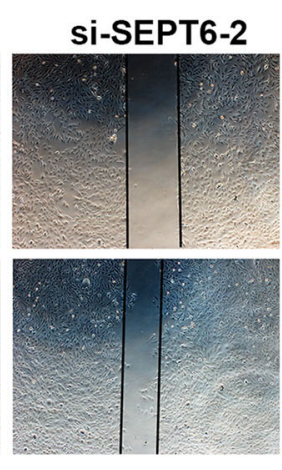
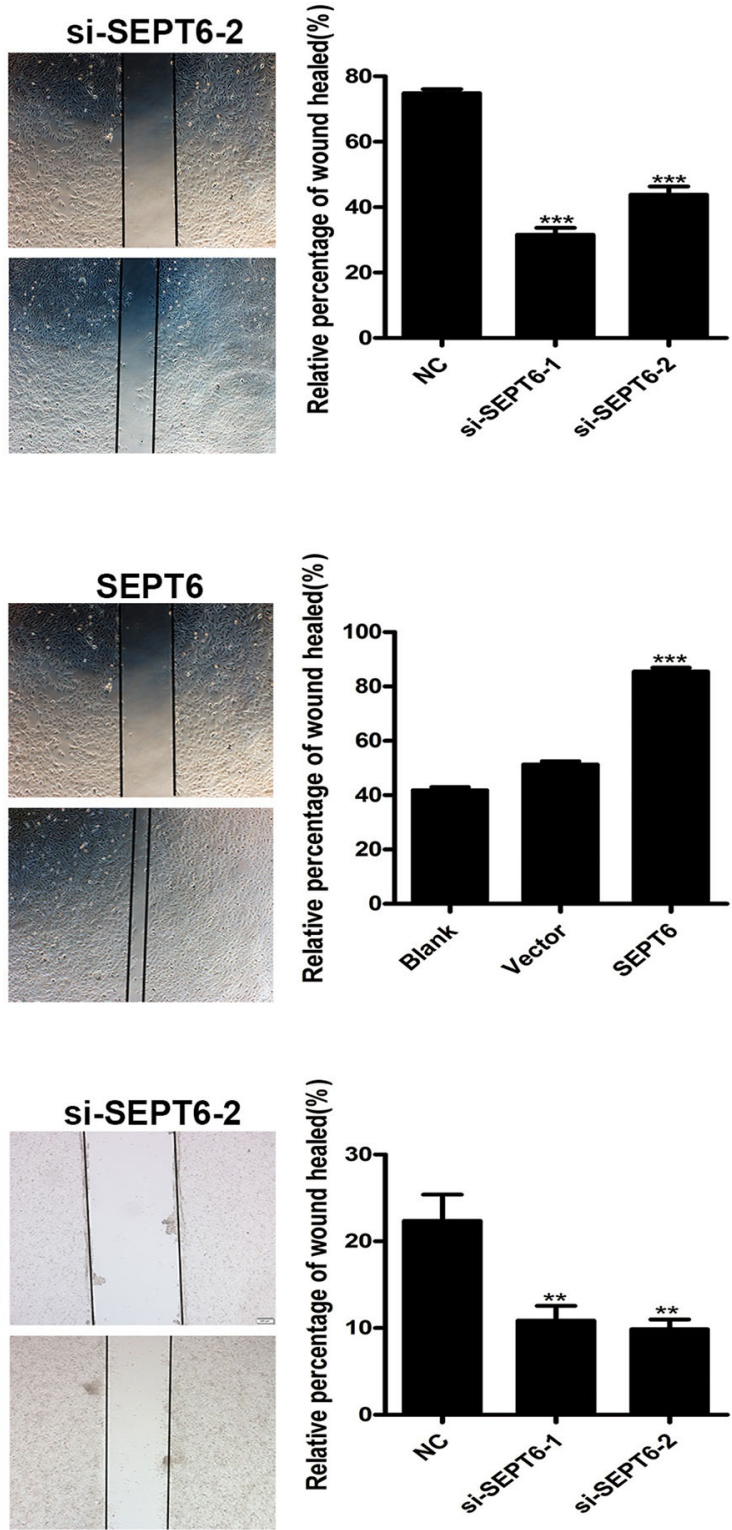

\section{응}

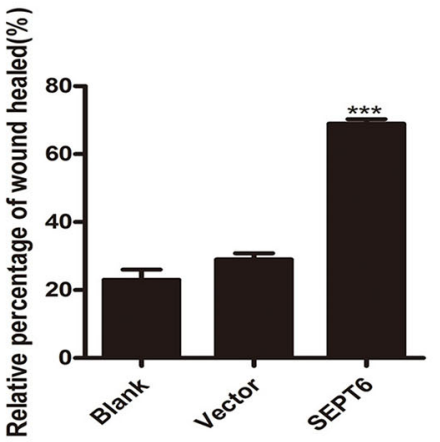



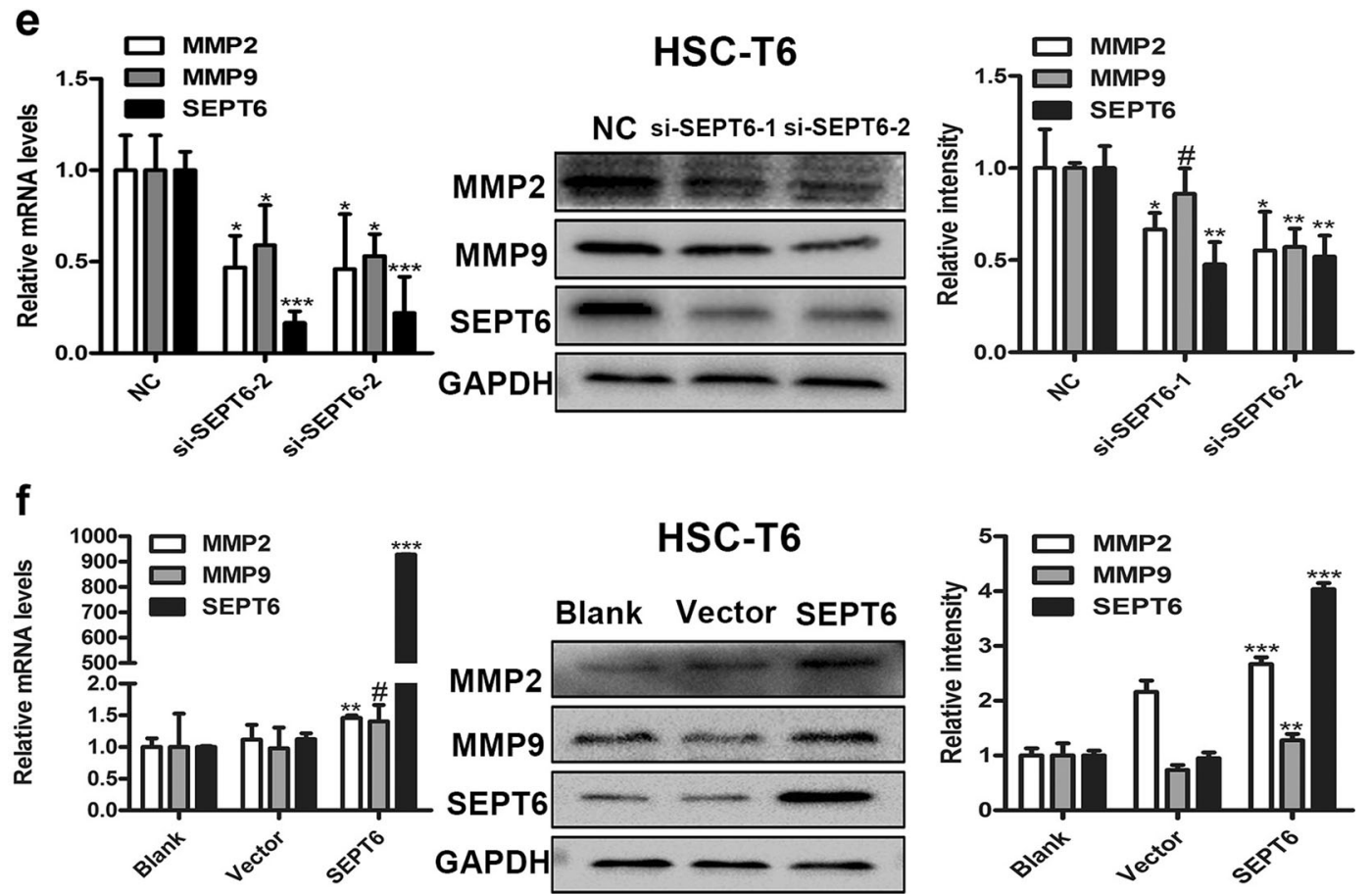

Fig. 6 SEPT6 promotes migration in HSCs in vitro. a-d The migration rates were quantified as a percentage of the wound-healed area using a wound-healing assay after SEPT6 expression was silenced in LX-2 cells a and HSC-T6 cells $\mathbf{c}$ or SEPT6 expression was enhanced in LX2 cells b and HSC-T6 cells $\mathbf{d}$. $(\times 40)$ Representative images of three independent experiments are shown. e, f Genes related to migration,

(Fig. 7c, d). These results suggest that SEPT6 promotes liver fibrosis likely by activating the MAPK and PI3K/AKT signaling pathways.

\section{Knockdown of SEPT6 ameliorates TAA-induced liver fibrosis in rats}

To explore the impact of SEPT6 on the progression of liver fibrosis in vivo, we silenced SEPT6 expression in a rat mode of TAA-induced liver fibrosis by injecting AdshSEPT6 through the tail vein. To determine the injection cycle for the adenovirus, we injected AdshSEPT6 through the tail vein, and the rats were sacrificed on the first day $(1 \mathrm{~d})$, fourth day $(4 \mathrm{~d})$, or seventh day $(7 \mathrm{~d})$. The realtime PCR and western blot results showed that compared with the NC group (PBS injected), SEPT6 expression decreased to the lowest level in the rat liver on the fourth day after the AdshSEPT6 injection (Fig. 8a). Thus, to maintain a low level of SEPT6 expression in the liver tissue, the adenovirus was injected twice a week for 2 weeks (Fig. 8b). During the model establishment, we observed that the NC group was generally in a good condition with a normal weight gain rate; whereas, the body weight gain of the rats in the model group (TAA injected) was slow and significantly lower than that in the normal group (Fig. 8c). including MMP2 and MMP9 were analysed by real-time PCR and western blot after SEPT6 expression was silenced $\mathbf{e}$ or enhanced $\mathbf{f}$ in HSC-T6 cells. GAPDH was used as a loading control. $* P<0.05, * * P$ $<0.01, * * * P<0.001$. siRNA vs. NC; SEPT6 vs. vector by Student's $t$ test

As shown in Fig. 8d, the liver of rats in the normal group (Fig. 8d-I) had a soft texture, a smooth surface and a sharp edge. The liver of the rats in the PBS control group (Fig. 8d-II) and AdGFP group (Fig. 8d-III) had coarse particles. However, the hepatic tissue in the AdshSEPT6 group (Fig. 8d-IV) was softer and smoother than that in the control group and AdGFP group. Though the liver morphology failed to return to normal throughout the process of TAA injection, we observed that compared with the AdGFP group, the SEPT6 silencing resulted in attenuated liver fibrosis as confirmed by H\&E, Masson trichrome and Sirius red staining (Fig. 8e). Furthermore, the AdshSEPT6 administration significantly reduced the hydroxyproline content (Fig. 8f). Consistent with the in vitro results, the mRNA and protein levels of $\alpha$-SMA, Cyclin D1, and MMP2 were significantly reduced after the AdshSEPT6 treatment compared with those after the AdGFP treatment, in contrast, the mRNA and protein levels of BAX increased significantly. (Fig. 9a, b). Compared with the AdGFP treatment, the expression of $\alpha$-SMA, COL1A1, and MMP2 was downregulated after the AdshSEPT6 treatment by immunohistochemistry (Fig. 9c). Moreover, the proliferative rat HSCs were confirmed by FITC-conjugated Ki67-staining, and the specific HSC marker $\alpha$-SMA was costained by cy3-labelled antibody. The results revealed that 

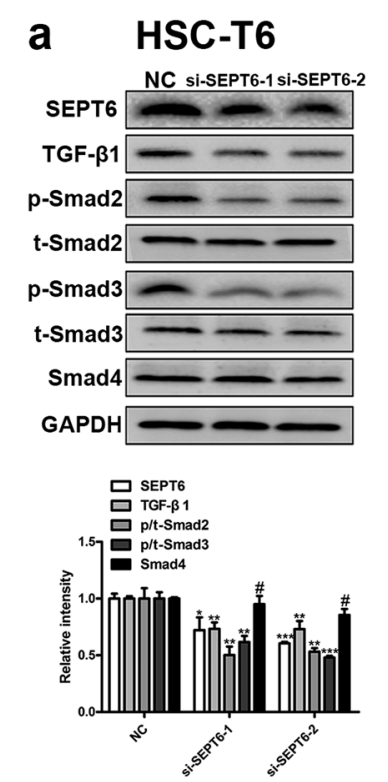
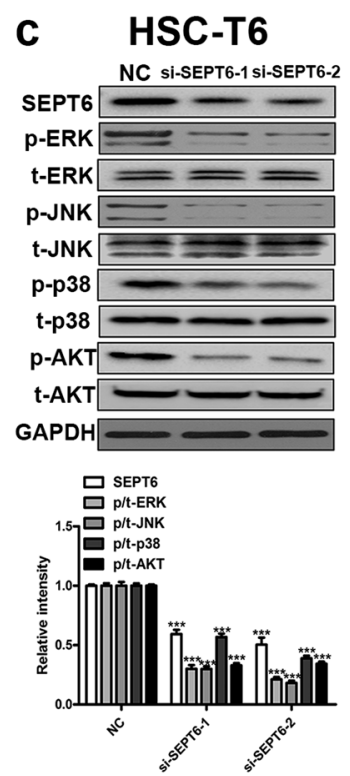
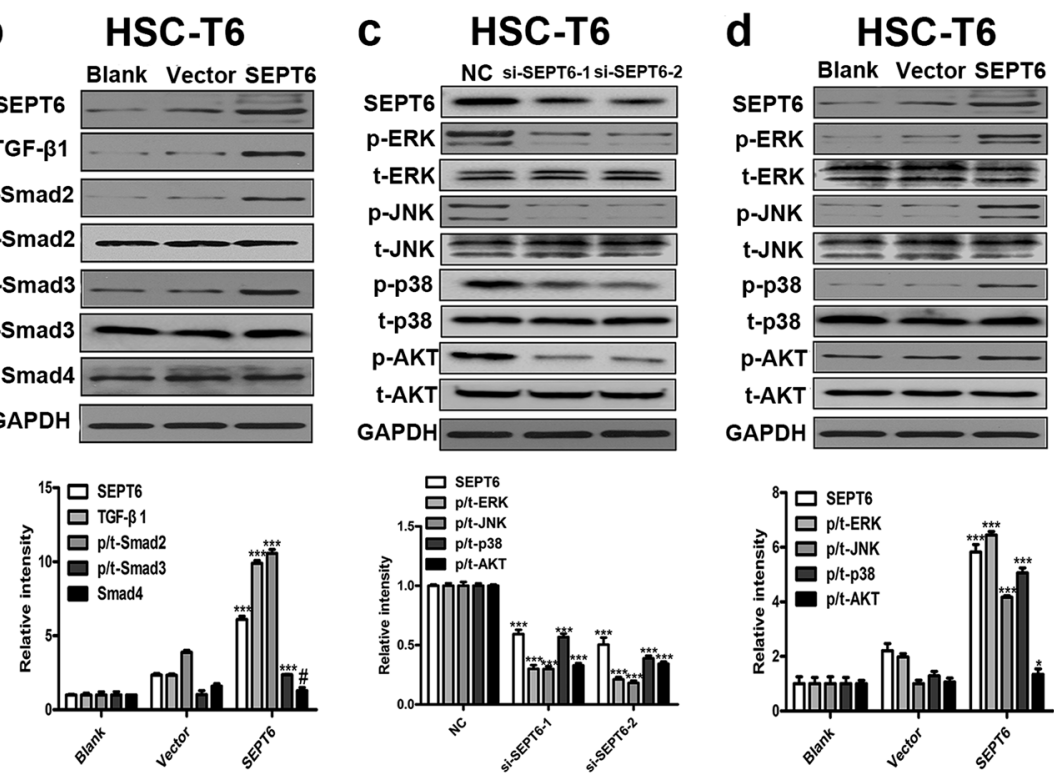

AKT, t-AKT, and GAPDH expression were assessed by western blot after SEPT6 expression was silenced $\mathbf{c}$ or enhanced $\mathbf{d}$ in HSC-T6 cells. GAPDH was used as a loading control. $* P<0.05$, $* * P<0.01$, *** $P<$ $0.001,{ }^{\#} P>0.05$. siRNA vs. NC; SEPT6 vs. vector by Student's $t$-test the enhanced HSC proliferation in the TAA-induced liver fibrosis model was significantly inhibited by the AdshSEPT6 administration and accompanied by the downregulation of $\alpha$-SMA (Fig. 9d). The apoptotic rat HSCs were detected using a FITC-conjugated TUNEL assay; the HSCs were also co-stained with cy3-labelled $\alpha$ SMA antibody, and the results revealed that the SEPT6 knockdown promoted rat HSC apoptosis, which was accompanied by the downregulation of $\alpha$-SMA (Fig. 9e). Taken together, the knockdown of SEPT6 ameliorated TAA-induced liver fibrosis in the rats.

\section{Discussion}

HSC activation plays a central role in the progression of liver fibrosis and is accompanied by enhanced ability of proliferation and migration and a decreased level of apoptosis $[3,33]$. Recently, increasing evidence has shown that the GTP-binding protein SEPT6 regulates cell proliferation, cell cycle progression, apoptosis, and migration in different diseases [22-24]. We detect the level of the entire septins member in healthy and fibrotic rat liver and find that the SEPT6 is elevated significantly. However, the expression and function of SEPT6 in HSCs and liver fibrosis remains unknown.

In this study, we utilized rat and cell models to investigate the effect of SEPT6 on liver fibrosis in vitro and in vivo. Here, we demonstrate for the first time that SEPT6 promotes HSC activation, proliferation, cell cycle progression, migration, and survival and that the blockade of SEPT6 can attenuate TAA-induced liver fibrosis in rats. We confirmed that SEPT6 expression was dramatically elevated in fibrotic human liver and TAA-induced fibrotic rat livers. The SEPT6 expression also increased in the activation of primary rat HSCs, LX-2 cells, and HSC-T6 cells. Moreover, the immunohistochemistry staining and the double immunofluorescence staining revealed that HSCs are a main source of the SEPT6, and SEPT6 localized both in the nucleus and cytoplasm. However, the localization of SEPT6 might be controversial, as described in process of $\mathrm{HCV}$ replication in Huh 5-15 cells, SEPT6 formed a protein complex with NS5b and hnRNPA1 through NS5b-SEPT6 and SEPT6- hnRNPA1 interactions, and the complex was important for HCV replication. In Huh 5-15 cells, it was also illustrated that, hnRNP A1 was initially located in the nucleus, then part of hnRNP A1 was redistributed into cytoplasm, and part remained in the nucleus, the relocalization of hnRNP A1 from the nucleus to the cytoplasm seemed to be specific to HCV replication, suggesting a cytoplasmic localization of SEPT6 in Huh 5-15 cells [36]. However, it is unknown whether SEPT6 can bind to hnRNP A1 which remained in nucleus, that is, it is unknown whether SEPT6 was also located in the nucleus. So we hypothesize that localization of SEPT6 might vary in different cell types, and this question needs further exploration.

To fully investigate the role of SEPT6 in HSC biological behaviors, we employed complementary methods, 


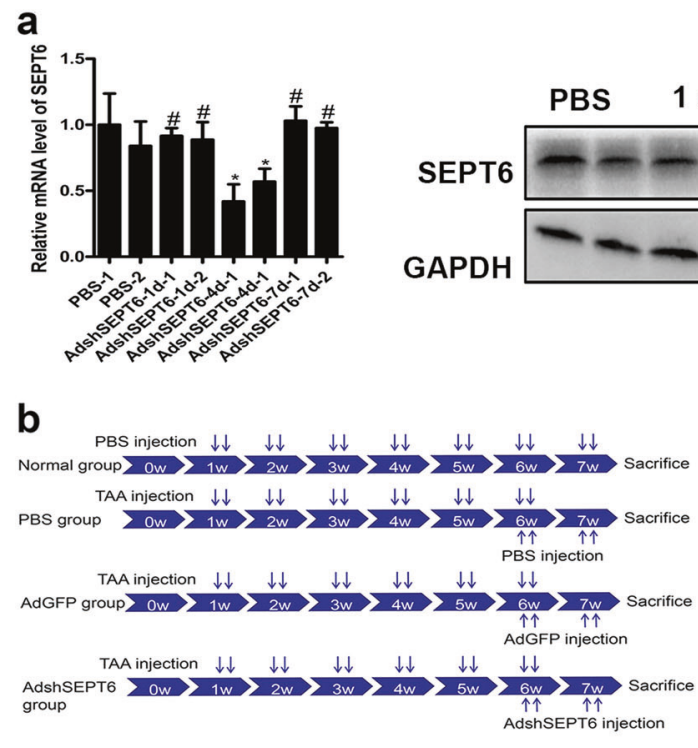

d
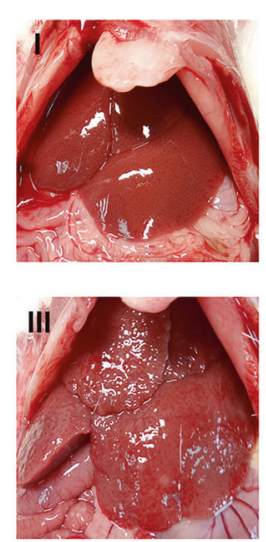

f

f Normal
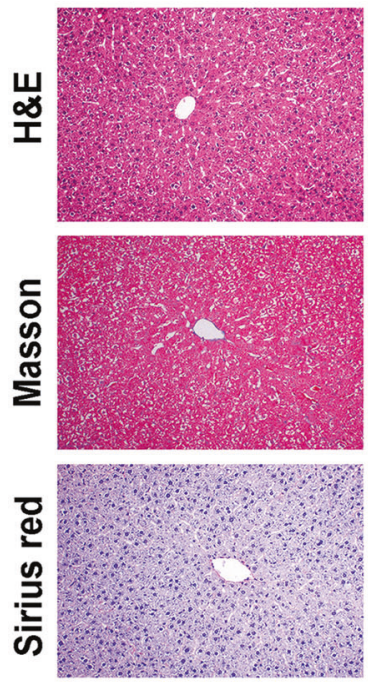

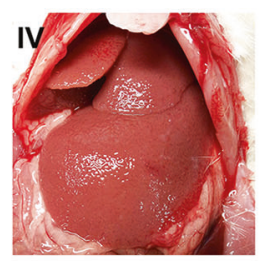

\section{PBS}
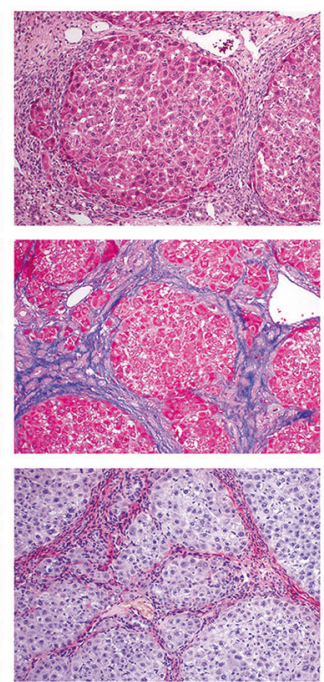

including gain- and loss-of-function analyses, to elucidate the role of SEPT6 in the activation, proliferation, cell cycle, apoptosis, and migration of HSCs. We confirmed that the
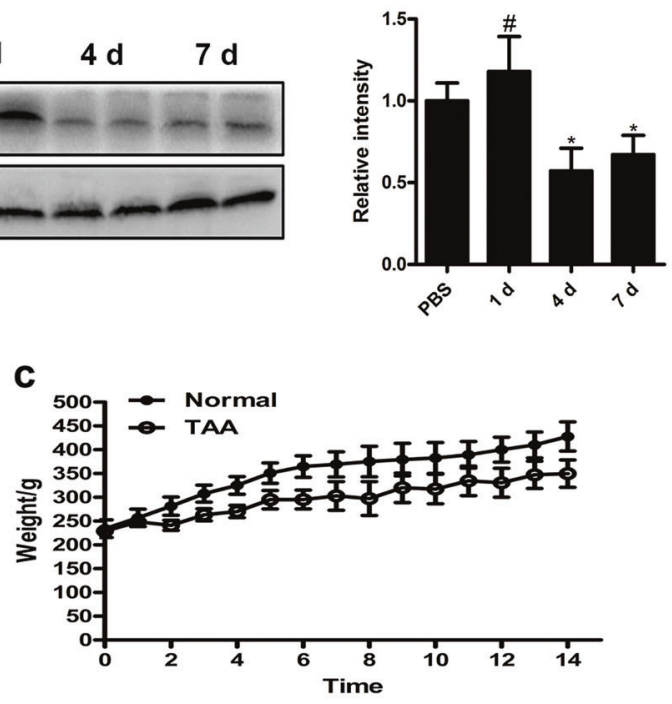

e

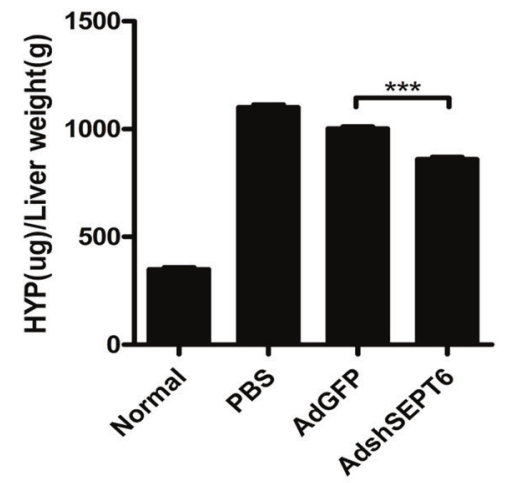

\section{AdGFP}

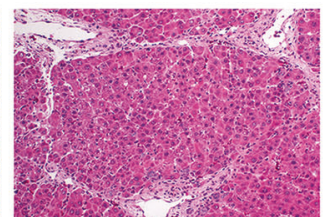

\section{AdshSEPT6}
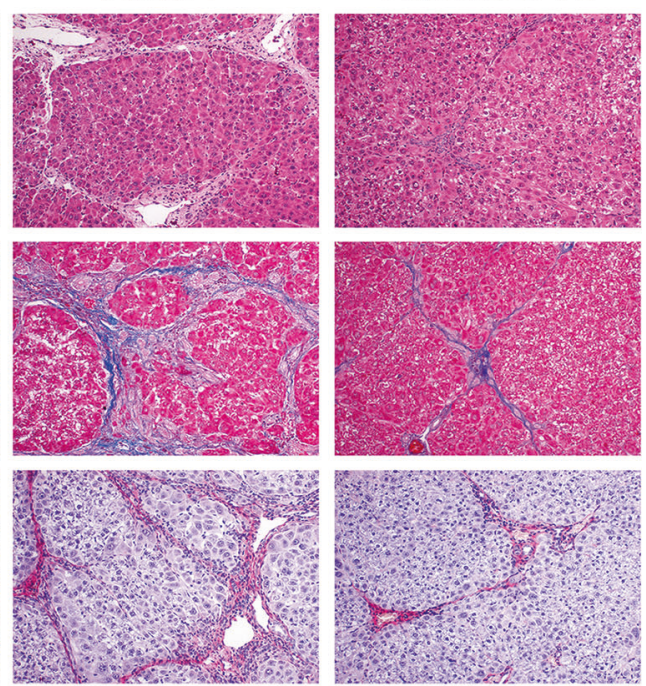

knockdown of SEPT6 by siRNA transfection significantly reduced $\alpha$-SMA and COL1A1 expression, while the overexpression of SEPT6 by plasmid transfection enhanced $\alpha$ - 
4 Fig. 8 Knockdown of SEPT6 ameliorates TAA-induced rat liver fibrosis. a SEPT6 expression was detected by real-time PCR and western blot on the $1 \mathrm{~d}, 4 \mathrm{~d}$, and $7 \mathrm{~d}$ after a tail vein injection of adshSEPT6 in normal rats; the control group was injected with PBS. b Animal model diagram. c Curve of the body weight gain in the model rats. $\mathbf{d}$ Liver appearance in different groups. I: Normal group; II: PBS group: III: AdGFP group; IV: AdshSEPT6 group. e Liver fibrosis was assessed by H\&E staining, Masson trichrome staining and Sirius red staining $(\times 100)$. f Hydroxyproline content in the livers from the AdshSEPT6- or AdGFP-treated rats in the TAA-induced fibrotic model was assessed $(n=10) * P<0.05, * * * P<0.001,{ }^{*} P>0.05 .1 \mathrm{~d}, 4$ d, 7 d vs. PBS; AdshSEPT6 vs. AdGFP by Student's $t$-test or one-way ANOVA

SMA and COL1A1 expression, suggesting that SEPT6 promotes HSC activation and ECM synthesis.

A recent study revealed that the knockdown of HBsAg inhibited HCC cell growth, and a microarray analysis revealed that SEPT6 decreased significantly after HBsAg inhibition; thus, we may hypothesize that SEPT6 promotes HCC proliferation [25]. Additionally, the SEPT2/6/7 complex can regulate cell cycle arrest in HeLa cells through the nuclear accumulation of NCK mediated by SOCS7 [23]. Our data provide evidence that the knockdown of SEPT6 in HSCs not only inhibits cell proliferation but also induces G1/S transition arrest and cell apoptosis. In contrast, the overexpression of SEPT6 promotes cell proliferation, G1/S transition progression and cell survival. Accordingly, we conclude that SEPT6 promotes proliferation in HSCs likely by promoting cell-cycle progression and inhibiting cell apoptosis. However, the role of SEPT6 in the G2/M transition was contradictory in LX-2 cells and HSC-T6 cells, which requires further elucidation. In liver fibrosis, activated HSCs can migrate vigorously, which is a distinctive property of activated HSCs [37]. The silencing of SEPT2, 6, and 7 causes actin cytoskeleton remodeling and changes in the cell shape, influencing actin-dependent processes, such as cell spreading in the ECM; thus, SEPT6 might regulate cell migration [23]. Consistent with this study, using wound-healing and Transwell migration assays, we demonstrated that the knockdown of SEPT6 significantly inhibits the migration capacity of LX-2 cells and HSC-T6 cells. In contrast, the enforced expression of SEPT6 dramatically induces LX-2 cell and HSC-T6 cell migration. Mechanistically, to induce migration, SEPT6 activated MMP2 and MMP9 expression in HSC-T6 cells.

Revealing the molecular mechanisms underlying the cell biological functions of SEPT6 is a crucial aim in our study. Some key signaling pathways, such as the TGF- $\beta 1 / \mathrm{Smad}$, MAPK, and PI3K/AKT signaling pathways, are known to be involved in the pathogenesis of liver fibrosis and substantially contribute to the regulation of the activation, proliferation, apoptosis, and migration of HSCs. However, whether the TGF- $\beta 1 /$ Smad, MAPK and PI3K/AKT signaling pathways are downstream targets of SEPT6 has not been clarified to date. We observed that in HSC-T6 cells the TGF- $\beta 1$ expression and the phosphorylation of Smad2, Smad3 were significantly downregulated by the SEPT6 knockdown and upregulated by SEPT6 overexpression. However, under both situations, the expression of total Smad2, Smad3, and Smad4 remained unchanged. Furthermore, blocking the TGF- $\beta 1 /$ Smad signaling pathway by SB431542 significantly decreased the expression of $\alpha$ SMA, Cyclin D1, BCL2, MMP2, MMP9, which were initially upregulated by SEPT6 overexpresion; in contrast, SB431542 enhanced the expression of BAX, which was initially downregulated by SEPT6 overexpresion. Intriguingly, as demonstrated in this study, the TGF- $\beta 1$ stimulation promoted SEPT6 expression in HSCs, thus, SEPT6 might form a positive feedback loop with TGF- $\beta 1$ in HSC activation and liver fibrosis. Furthermore, p-ERK, p-JNK, p-p38, p-AKT were downregulated by the knockdown of SEPT6 and upregulated by the SEPT6 overexpression. However, the expression of total ERK, JNK, p38, and AKT remained unchanged despite the shift in the expression of SEPT6. Conclusively, SEPT6 may regulate cell behavior through the TGF- $\beta 1 /$ Smad, MAPK, and PI3K/AKT signaling pathways in HSCs. To the best of our knowledge, the crosstalk between TGF- $\beta 1 /$ Smad, MAPK, and PI3K/AKT is complicated. It has been confirmed that MAPK and PI3K/ AKT are downstream components of TGF- $\beta 1$ signaling in HSCs. In contrast, MAPK mediates TGF- $\beta 1$ signaling through Smad3 phosphorylation [35], while the inhibition of PI3K/AKT can also reduce TGF- $\beta 1$ expression [9]. The mechanisms by which SEPT6 influences the crosstalk and the specific pathway that exerts the predominant effect need further study.

Finally, animal experiments were performed to confirm the role of SEPT6 in vivo. In this study, we demonstrated that adenovirus-mediated knockdown of SEPT6 significantly attenuates the progression of TAAinduced liver fibrosis in rats. Compared with the AdGFP injection, the adshSEPT6 significantly mitigated ECM deposition and decreased the expression of $\alpha$-SMA, which is a marker of HSC activation, indicating that SEPT6 may attenuate liver fibrosis by inducing HSC deactivation. Consistent with the in vitro study, SEPT6 knockdown reduced HSC proliferation and induced HSC apoptosis, which was separately confirmed by Ki67-staining (costained with $\alpha$-SMA) and a TUNEL assay (co-stained with $\alpha$-SMA). Moreover, the SEPT6 inhibition in vivo reduced Cyclin D1 expression and enhanced BAX expression. During liver fibrogenesis, HSC migratory capacity is tremendously enhanced, which is accompanied by the upregulation of MMPs, which contributes to the degradation of the ECM and partially accounts for the enhanced migratory capacity of HSCs [38]. Our in vivo study showed that 
SEPT6 knockdown significantly reduces MMP2 expression, confirming the pro-migration effect of SEPT6 demonstrated in vitro.
However, contrasting results have been found in prostate cancer in which SEPT6 was significantly decreased in prostate cancer tissue and cell lines compared with that in
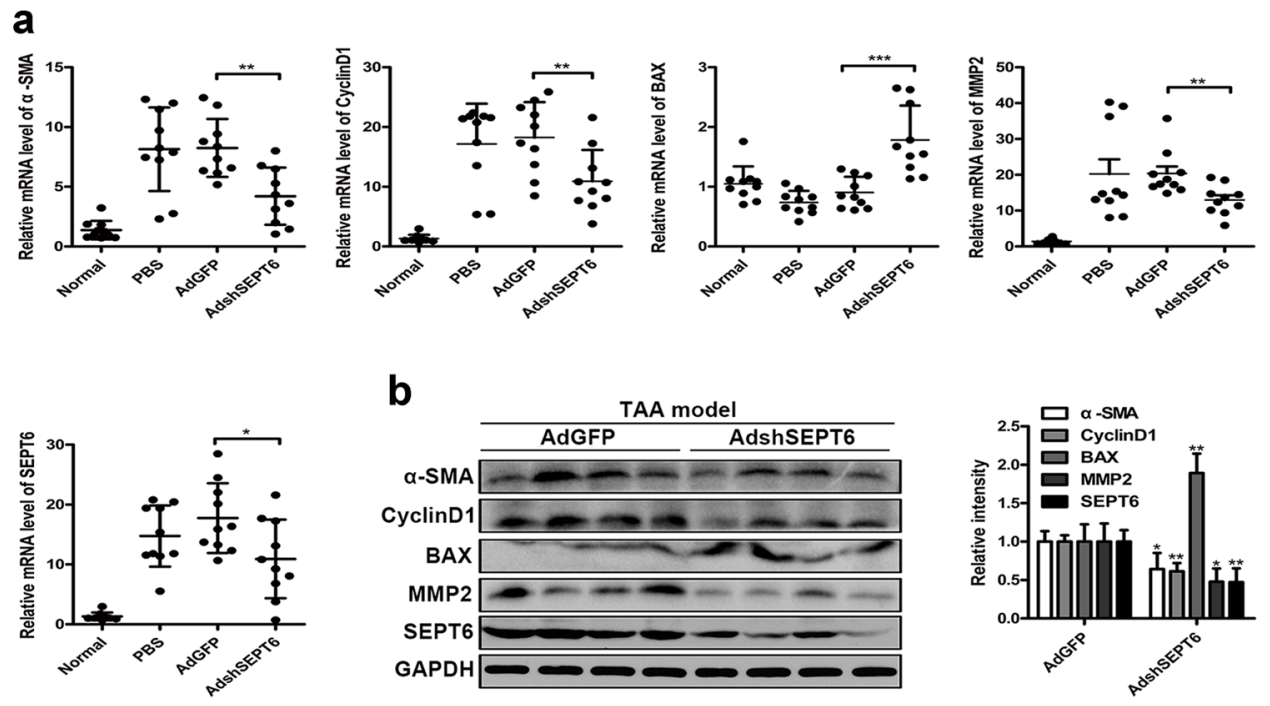

b
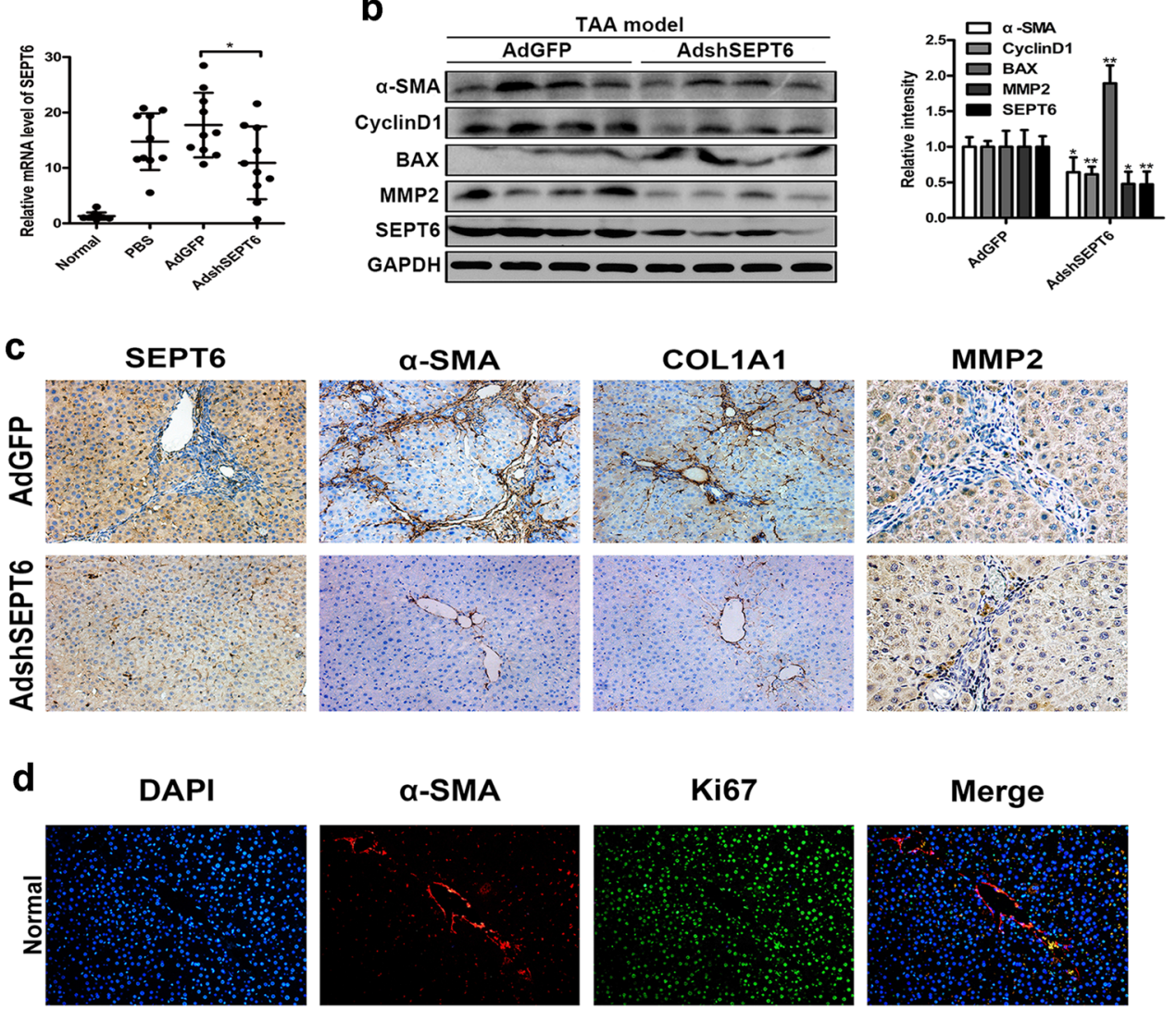

$\alpha-S M A$

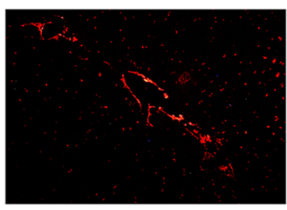

Ki67

Merge
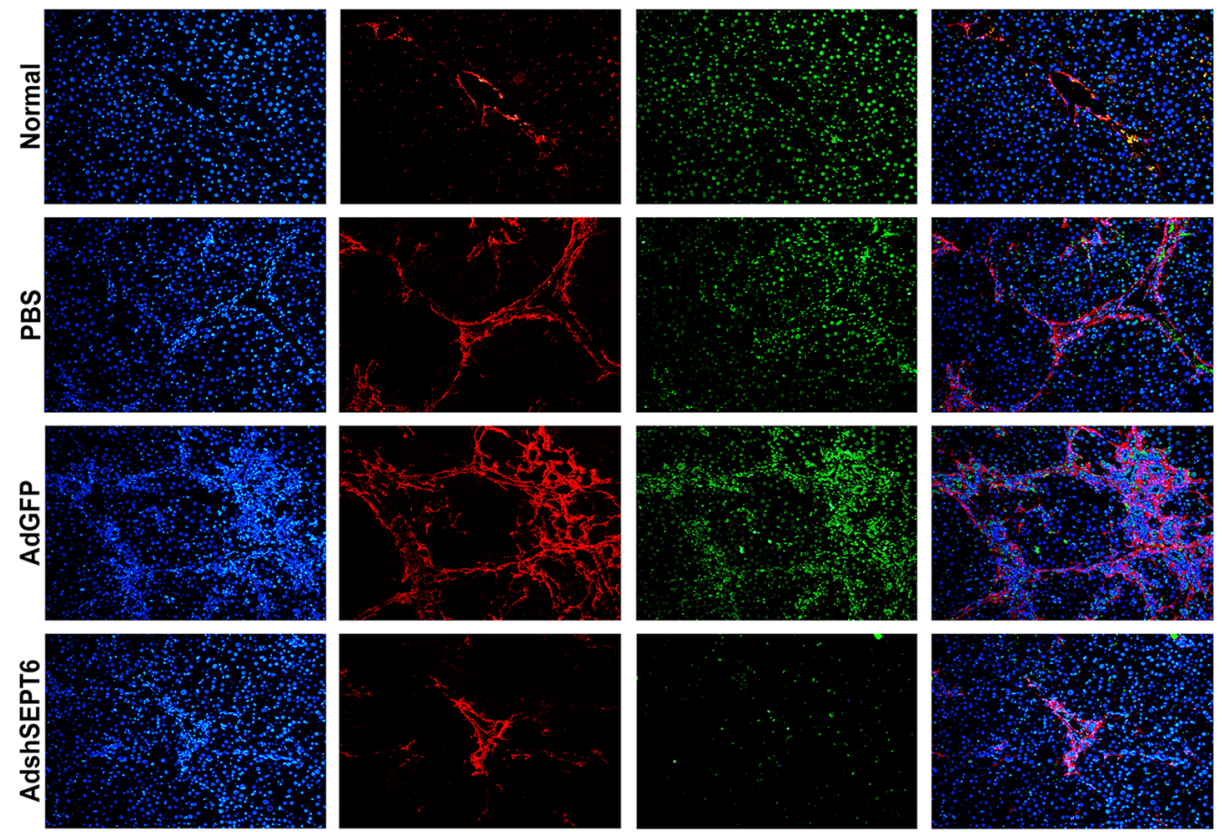
e
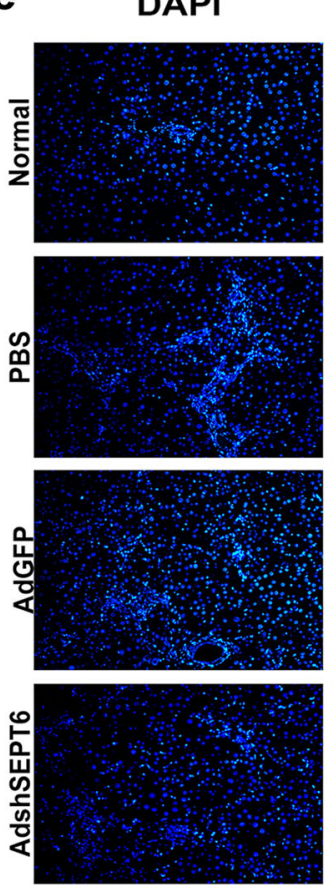

$\alpha-S M A$
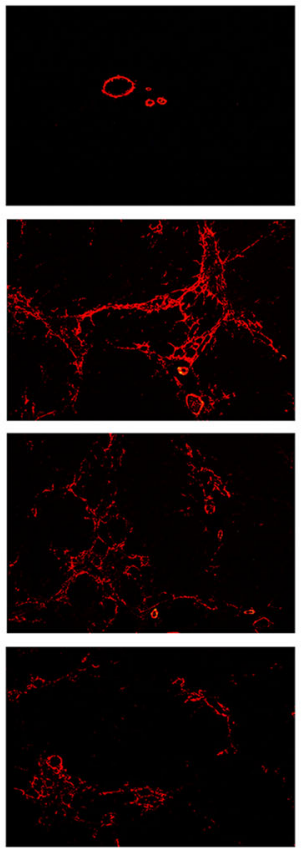

Tunel
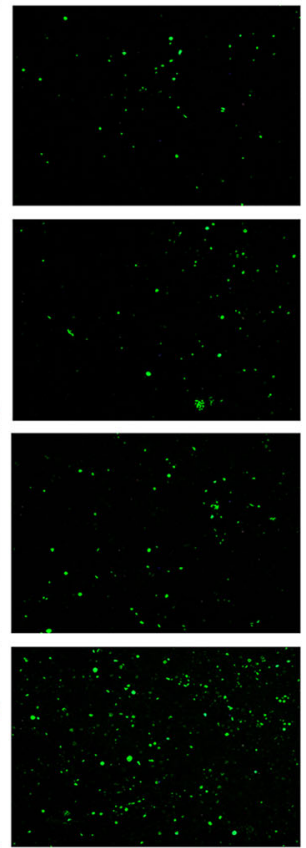

Merge
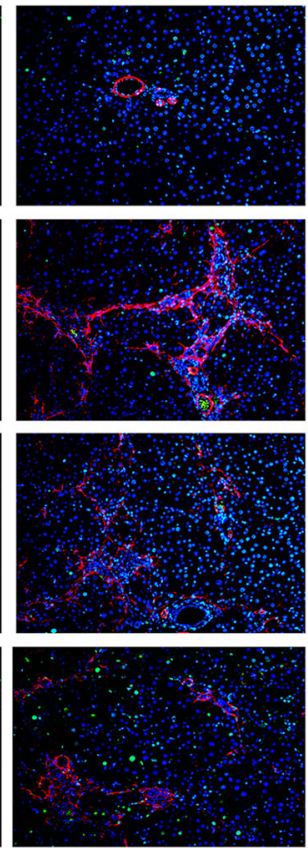

Fig. 9 Knockdown of SEPT6 reduces the activation, proliferation, migration, and survival of HSCs in vivo. a, b AdshSEPT6 or AdGFP was injected into rats 6 weeks after the administration of TAA. After 2 weeks, the level of $\alpha$-SMA, Cyclin D1, BAX, MMP2, and SEPT6 in the fibrotic livers was analysed by real-time PCR $\mathbf{a}(n=10)$ and western blot $\mathbf{b}(n=4)$. $\mathbf{c}$ Immunohistochemistry was used to detect the expression of SEPT6, $\alpha$-SMA, COL1A1, and MMP2. $(\times 200)(n=3)$. d Proliferative cells were immunostained with anti-Ki67-antibodies (green) and presence of myofibroblasts was determined by Cy3-conjugated anti- $\alpha$-SMA immunostaining (red). $(\times 100)(n=5)$. Representative images are shown. e Nuclei of apoptotic cells were FITC (green) labelled using a TUNEL assay, while the cytoskeleton of the myofibroblasts was stained by red immunofluorescence by means of Cy3-conjugated anti- $\alpha$-SMA antibodies. $(\times 100)(n=5)$ Representative images are shown. $* P<0.05$, $* * P<0.01, * * * P<$ 0.001 , AdshSEPT6 vs. AdGFP by Student's $t$-test

adjacent non-tumor tissues and the non-cancer cell line RWPE-1. Notably, the SEPT6 knockdown significantly inhibited apoptosis in a prostate cancer cell line and promoted its migration and invasion [15]. A potential explanation is that the genetic specificity of cells critically determines the function of SEPT6. However, the molecular and cellular mechanisms underlying such events require further investigation. Furthermore, different septin members might have different biological function. It has been reported that SEPT4 and SEPT9 were remarkably downregulated in HSC activation and liver fibrosis; loss-ofSEPT4 exacerbated liver fibrosis [26], and SEPT9 contributed to alleviate liver fibrosis partially through promoting activated HSCs apoptosis [27]. Taken together, we demonstrated for the first time that SEPT6 promotes HSCs activation, proliferation, cell cycle progression, migration, and survival, likely by activating the TGF- $\beta 1 /$ Smad, MAPK, and PI3K/AKT signaling pathways. Furthermore, our in vivo study demonstrated that SEPT6 inhibition attenuates TAA-induced liver fibrosis in rats. Collectively, the evidences suggest the important role of SEPT6 in HSCs activation and fibrosis.
Acknowledgements This study was supported by the National Natural Science Foundation of China (No. 81272657, No. 81572422).

\section{Compliance with ethical standards}

Conflict of interest The authors declare that they have no conflict of interest.

\section{References}

1. Hernandez-Gea V, Friedman SL. Pathogenesis of liver fibrosis. Annu Rev Pathol. 2011;6:425-56.

2. Puche JE, Saiman Y, Friedman SL. Hepatic stellate cells and liver fibrosis. Compr Physiol. 2013;3:1473-92.

3. Gonzalez SA, Fiel MI, Sauk J, et al. Inverse association between hepatic stellate cell apoptosis and fibrosis in chronic hepatitis $\mathrm{C}$ virus infection. J Viral Hepat. 2009;16:141-8.

4. Krizhanovsky V, Yon M, Dickins RA, et al. Senescence of activated stellate cells limits liver fibrosis. Cell. 2008;134:657-67.

5. Inagaki Y, Okazaki I. Emerging insights into transforming growth factor beta Smad signal in hepatic fibrogenesis. Gut. 2007;56:284-92.

6. Marra F, Delogu W, Petrai I, et al. Differential requirement of members of the MAPK family for CCL2 expression by hepatic stellate cells. Am J Physiol Gastrointest Liver Physiol. 2004;287: G18-G26. 
7. Johnson GL, Lapadat R. Mitogen-activated protein kinase pathways mediated by ERK, JNK, and p38 protein kinases. Science. 2002;298:1911-2.

8. Kluwe J, Pradere JP, Gwak GY, et al. Modulation of hepatic fibrosis by c-Jun-N-terminal kinase inhibition. Gastroenterology. 2010;138:347-59.

9. Son G, Hines IN, Lindquist J, et al. Inhibition of phosphatidylinositol 3-kinase signaling in hepatic stellate cells blocks the progression of hepatic fibrosis. Hepatology. 2009;50:1512-23.

10. Longtine MS, DeMarini DJ, Valencik ML, et al. The septins: roles in cytokinesis and other processes. Curr Opin Cell Biol. 1996;8:106-19.

11. Hall PA, Jung K, Hillan KJ, et al. Expression profiling the human septin gene family. J Pathol. 2005;206:269-78.

12. Pan F, Malmberg RL, Momany M. Analysis of septins across kingdoms reveals orthology and new motifs. BMC Evol Biol. 2007;7:103.

13. Spiliotis ET, Kinoshita M, Nelson WJ. A mitotic septin scaffold required for mammalian chromosome congression and segregation. Science. 2005;307:1781-5.

14. Sandrock K, Bartsch I, Blaser S, et al. Characterization of human septin interactions. Biol Chem. 2011;392:751-61.

15. Zhou J, Lu S, Yang S, et al. MicroRNA-127 post-transcriptionally downregulates Sept7 and suppresses cell growth in hepatocellular carcinoma cells. Cell Physiol Biochem. 2014;33:1537-46.

16. Zhang N, Liu L, Fan N, et al. The requirement of SEPT2 and SEPT7 for migration and invasion in human breast cancer via MEK/ERK activation. Oncotarget. 2016;7:61587-61600.

17. Amir S, Golan M, Mabjeesh NJ. Targeted knockdown of SEPT9_v1 inhibits tumor growth and angiogenesis of human prostate cancer cells concomitant with disruption of hypoxiainducible factor-1 pathway. Mol Cancer Res. 2010;8:643-52.

18. Cerveira N, Bizarro S, Teixeira MR. MLL-SEPTIN gene fusions in hematological malignancies. Biol Chem. 2011;392:713-24.

19. Cho SJ, Lee H, Dutta S, et al. Septin 6 regulates the cytoarchitecture of neurons through localization at dendritic branch points and bases of protrusions. Mol Cells. 2011;32:89-98.

20. Moon IS, Lee H, Walikonis RS. Septin 6 localizes to microtubules in neuronal dendrites. Cytotechnology. 2013;65:179-86.

21. Hu J, Bai X, Bowen JR, et al. Septin-driven coordination of actin and microtubule remodeling regulates the collateral branching of axons. Curr Biol. 2012;22:1109-15.

22. Kremer BE, Haystead T, Macara IG. Mammalian septins regulate microtubule stability through interaction with the microtubulebinding protein MAP4. Mol Biol Cell. 2005;16:4648-59.

23. Kremer BE, Adang LA, Macara IG. Septins regulate actin organization and cell-cycle arrest through nuclear accumulation of NCK mediated by SOCS7. Cell. 2007;130:837-50.
24. Wei Y, Yang J, Yi L, et al. MiR-223-3p targeting SEPT6 promotes the biological behavior of prostate cancer. Sci Rep. 2014;4:7546.

25. Xiangji L, Feng X, Qingbao C, et al. Knockdown of HBV surface antigen gene expression by a lentiviral microRNA-based system inhibits HBV replication and HCC growth. J Viral Hepat. 2011;18:653-60.

26. Iwaisako K, Hatano E, Taura K, et al. Loss of Sept 4 exacerbates liver fibrosis through the dysregulation of hepatic stellate cells. J Hepatol. 2008;49:768-78.

27. Wu Y, Bu F, Yu H, et al. Methylation of Septin9 mediated by DNMT3a enhances hepatic stellate cells activation and liver fibrogenesis. Toxicol Appl Pharmacol. 2017;315:35-49.

28. Trebicka J, Hennenberg M, Odenthal M, et al. Atorvastatin attenuates hepatic fibrosis in rats after bile duct ligation via decreased turnover of hepatic stellate cells. J Hepatol. 2010;53:702-12.

29. Lin J, Chen A. Activation of peroxisome proliferator-activated receptor-gamma by curcumin blocks the signaling pathways for PDGF and EGF in hepatic stellate cells. Lab Invest. 2008;88:529-40.

30. He J, Gong J, Ding Q, et al. Suppressive effect of SATB1 on hepatic stellate cell activation and liver fibrosis in rats. FEBS Lett. 2015;589:1359-68.

31. Gressner AM, Weiskirchen R, Breitkopf K, et al. Roles of TGFbeta in hepatic fibrosis. Front Biosci. 2002;7:d793-807.

32. Kennedy LL, Meng F, Venter JK, et al. Knockout of microRNA21 reduces biliary hyperplasia and liver fibrosis in cholestatic bile duct ligated mice. Lab Invest. 2016;96:1256-67.

33. Uyama N, Iimuro Y, Kawada N, et al. Fascin, a novel marker of human hepatic stellate cells, may regulate their proliferation, migration, and collagen gene expression through the FAK-PI3KAkt pathway. Lab Invest. 2012;92:57-71.

34. Derynck R, Zhang YE. Smad-dependent and Smad-independent pathways in TGF-beta family signaling. Nature. 2003;425:577-84.

35. Furukawa F, Matsuzaki K, Mori S, et al. p38 MAPK mediates fibrogenic signal through $\mathrm{Smad} 3$ phosphorylation in rat myofibroblasts. Hepatology. 2003;38:879-89.

36. Kim CS, Seol SK, Song OK, et al. An RNA-binding protein, hnRNP A1, and a scaffold protein, septin 6 , facilitate hepatitis C virus replication. J Virol. 2007;81:3852-65.

37. Friedman SL. Evolving challenges in hepatic fibrosis. Nat Rev Gastroenterol Hepatol. 2010;7:425-36.

38. Galli A, Svegliati-Baroni G, Ceni E, et al. Oxidative stress stimulates proliferation and invasiveness of hepatic stellate cells via a MMP2-mediated mechanism. Hepatology. 2005; 41:1074-84. 